
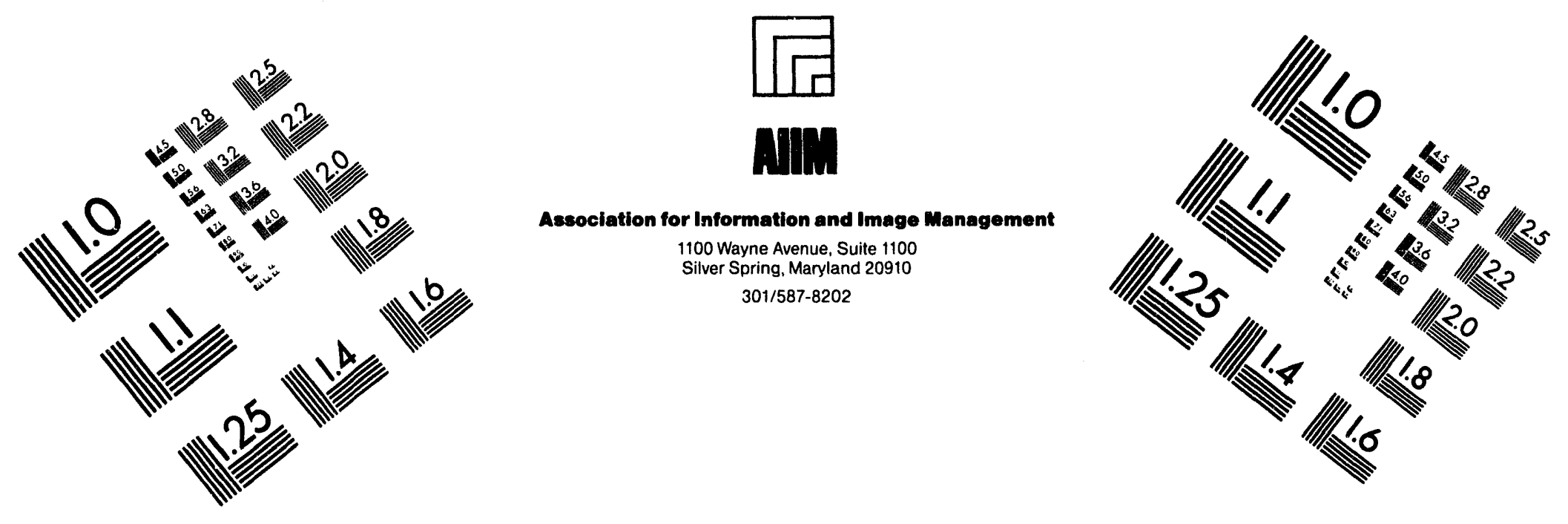

\title{
Centimeter
}

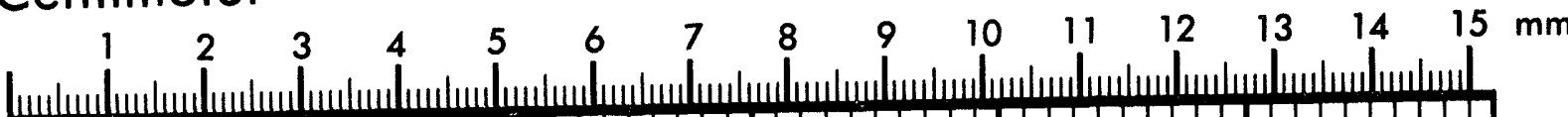

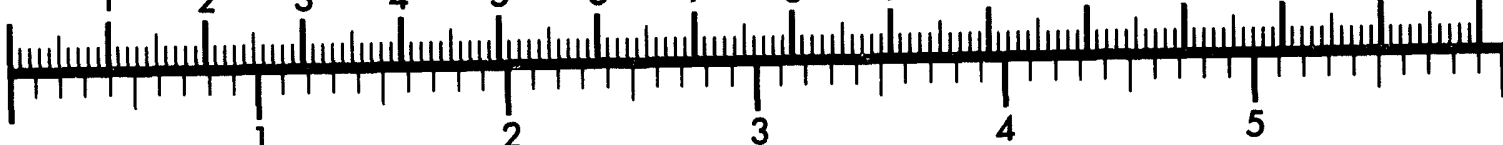
Inches
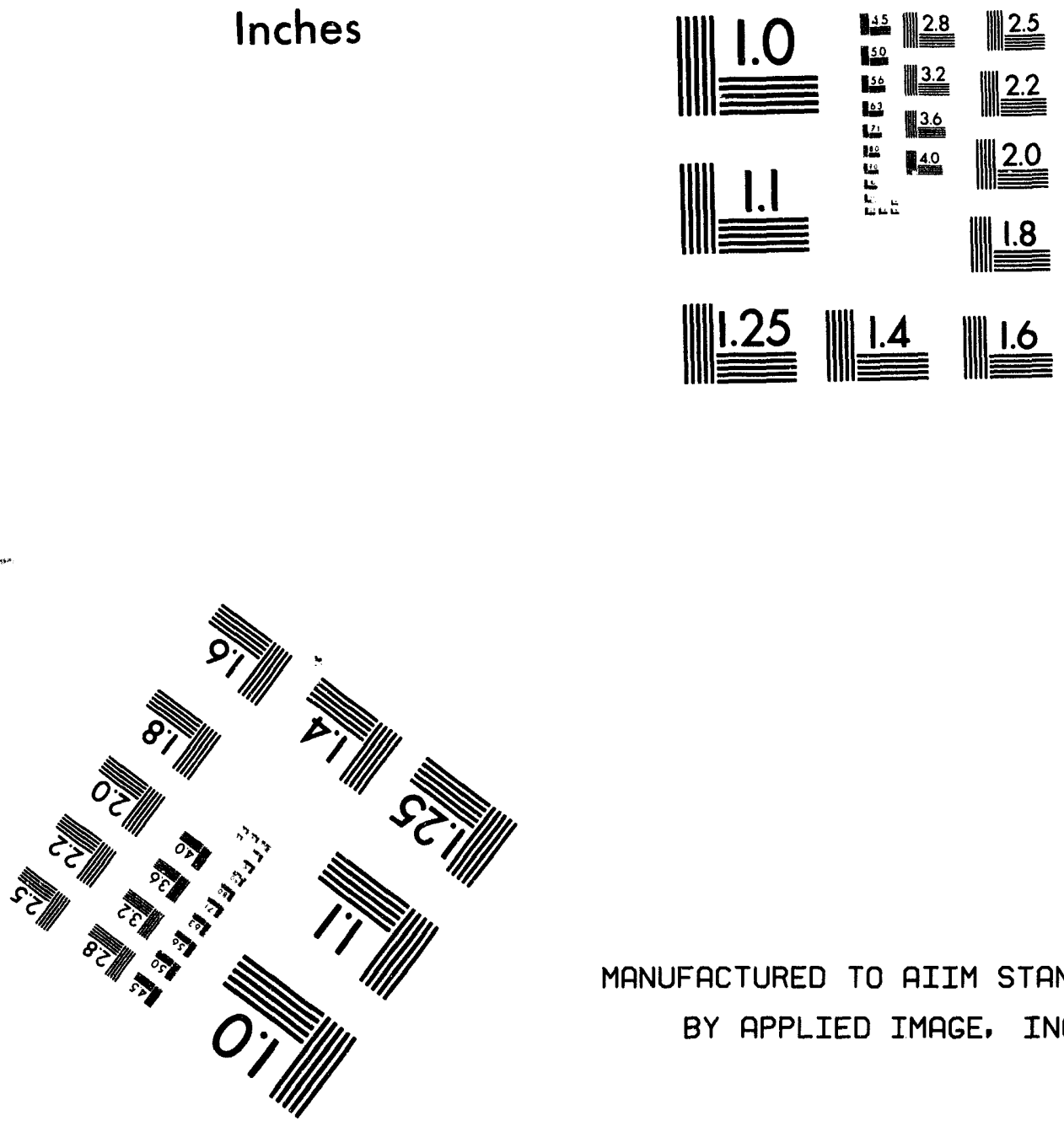

MANUFACTURED TO AIIM STANDARDS

BY APPLIED IMAGE, INC.

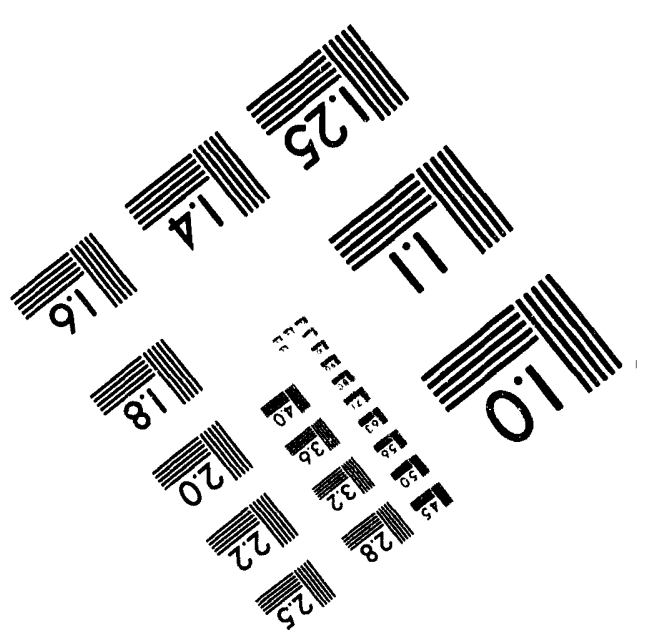



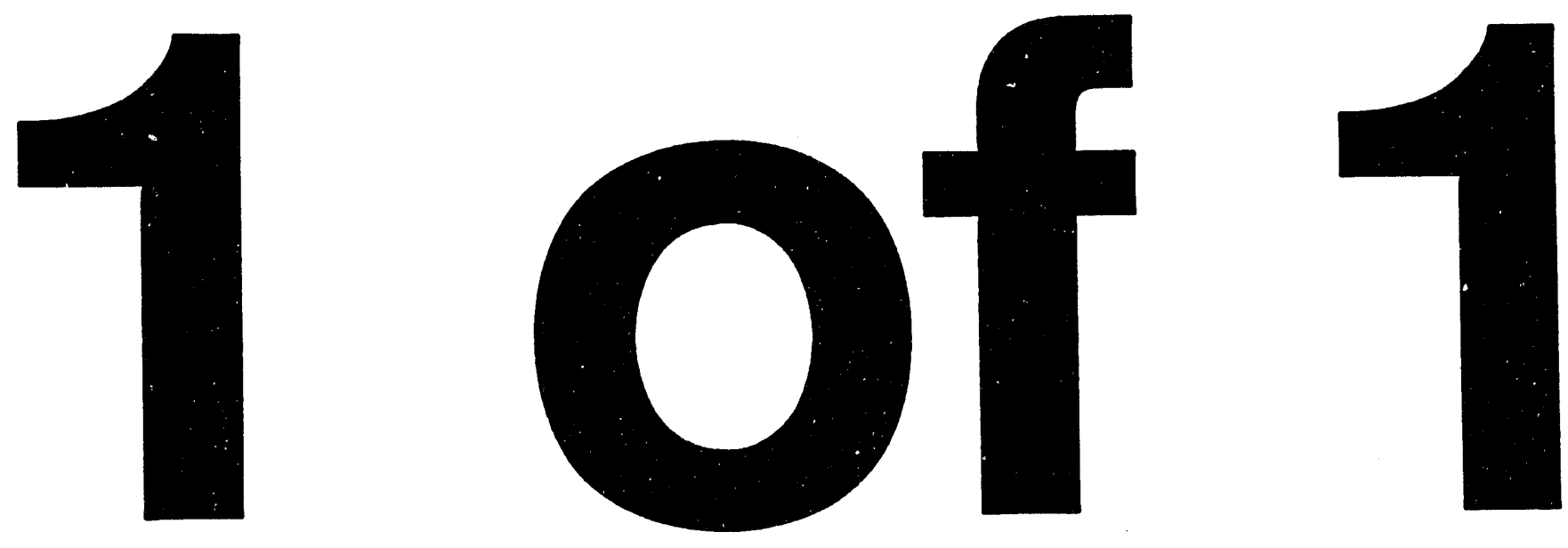


\section{Applications of a New Magnetic Monitoring Technique to in situ Evaluation of Fatigue Damage in Ferrous Components}

Manuscript Completed: May 1994

Date Published: June 1994

Prepared by

D. C. Jiles, S. B. Biner, M. R. Govindaraju, Z. J. Chen

Center for NDE

Iowa State University

Ames, Iowa 50011

\section{Prepared for}

Division of Engineering

Office of Nuclear Regulatory Research

U.S. Nuclear Regulatory Commission

Washington, DC 20555-0001

\section{MASTER}




\begin{abstract}
The work undertaken in this project consisted of research into the use of magnetic inspection methods for the estimation of fatigue life of nuclear pressure vessel steel. The rationale for this work was that the mechanical and magnetic properties of ferromagnetic materials are closely interrelated, and therefore the measurements of the magnetic properties could be used to monitor the evolution of fatigue damage in these specimens as they were subjected to cyclic loading.

The results of the work have shown that it is possible to monitor the fatigue damage nondestructively by magnetic techniques. For

example, in load-controlled high-cycle fatigue tests, it has been found that the plastic strain and coercivity accumulate logarithmically during the fatigue process. Thus a quantitative relationship between coercivity and the number of fatigue cycles could be established based on two ennpirical coefficients, which can be determined from the test conditions and material properties. Also it was found that prediction of the onset of fatigue failure in steels was possible under certain conditions. In strain-controlled low cycle fatigue, critical changes in Barkhausen emissions, coercivity and hysteresis loss occurred in the last ten to twenty percent of fatigue life.
\end{abstract}




\section{Contents}

Page

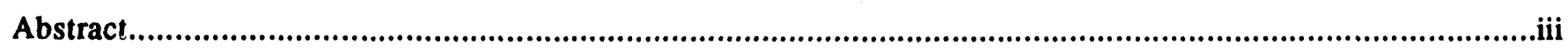

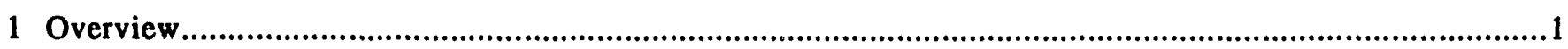

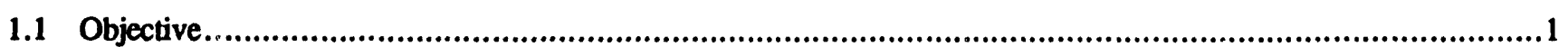

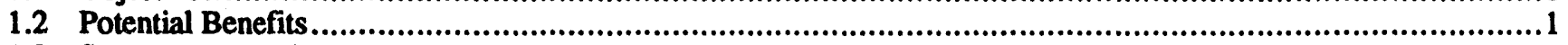

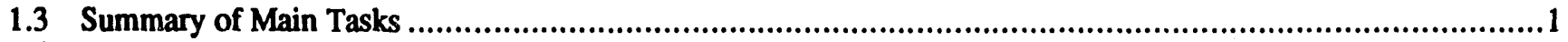

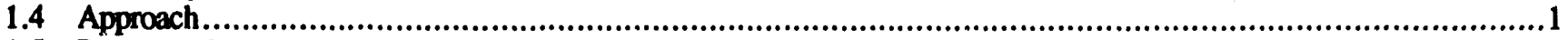

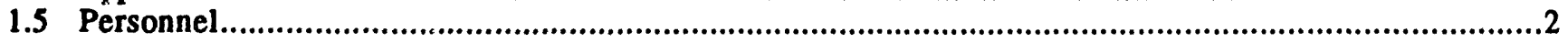

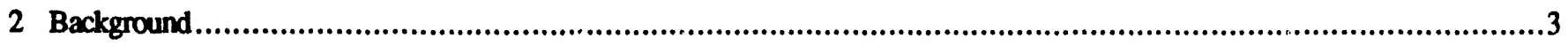

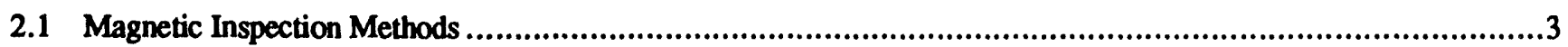

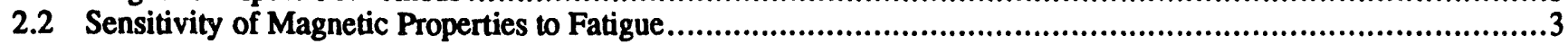

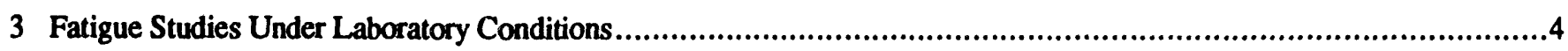

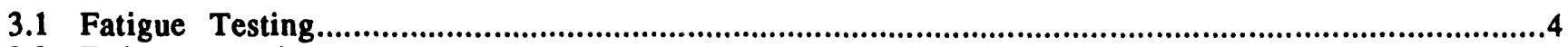

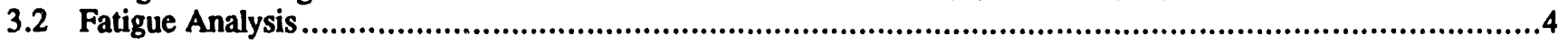

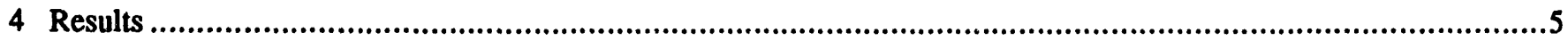

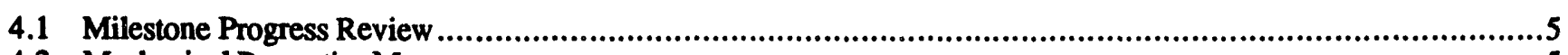

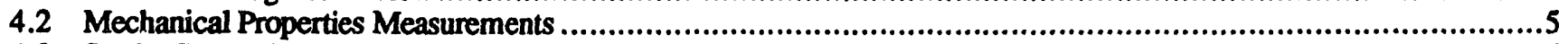

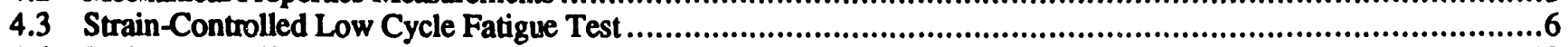

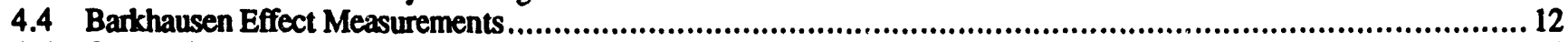

4.5 Comparison of Magnetic Hysteresis Measurement and Barkhausen Effect Measurement. ..................................... 14

4.6 Metallographic Studies ............................................................................................................ 15

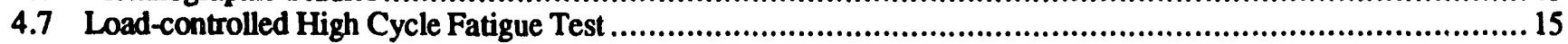

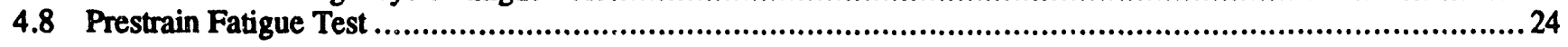

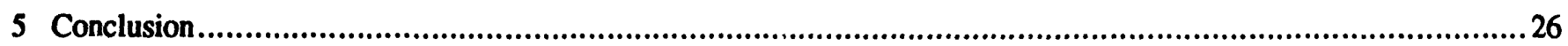

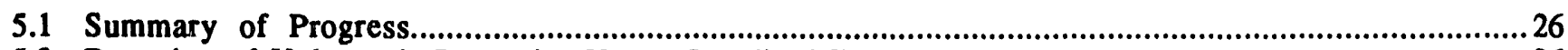

5.2 Detection of Volumetric Properties Versus Localized Flaws.....................................................................26

5.3 Detection of Strain-Controlled Fatigue and Load-Controlled Fatigue................................................................26

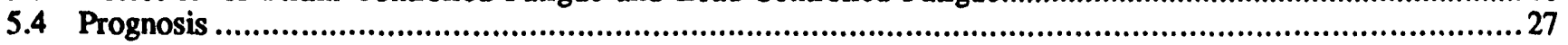

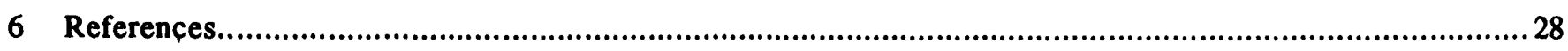

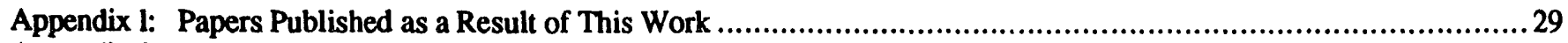

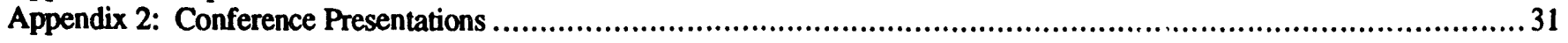




\section{Figures}

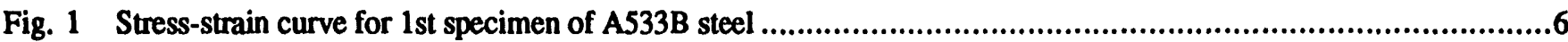

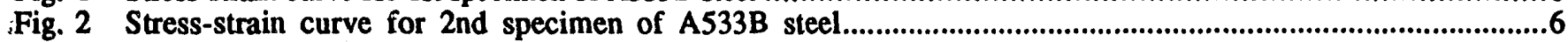

Fig. 3 Variation of tensile and compressive load with expended fatigue life for Sample EP05 with fatigue strain

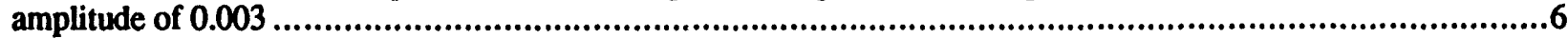

Fig. 4 Load-strain curve for Sample EP05 at fatigue cycle \# 5..................................................................................

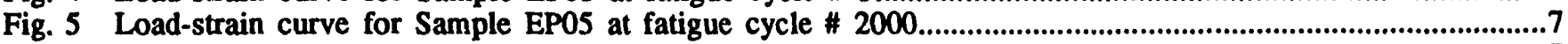

Fig. 6 Load-strain curve for Sample EP05 at fatigue cycle \# 4000

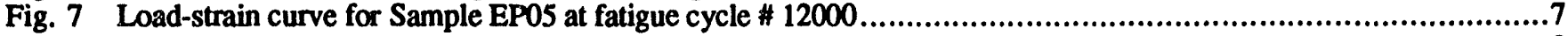

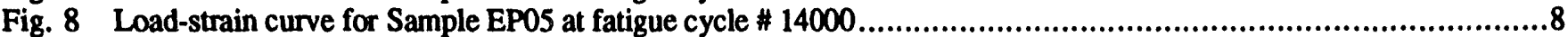

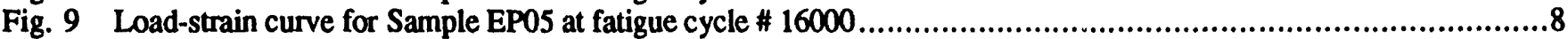

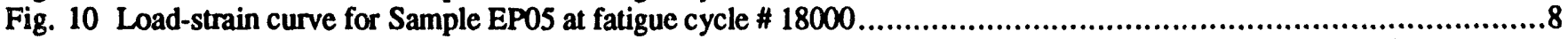

Fig. 11 Variation of tensile and compressive load with expended fatigue life for Sample MP03 with fatigue strain

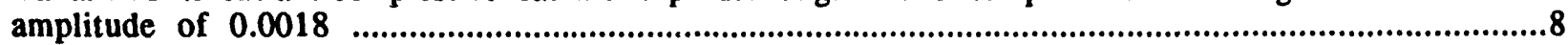

Fig. 12 Load-strain curve for Sample MP03 at fatigue cycle \# 5.............................................................................9

Fig. 13 Load-strain curve for Sample MP03 at fatigue cycle \# 10000, area within loop indicating micro-yielding of

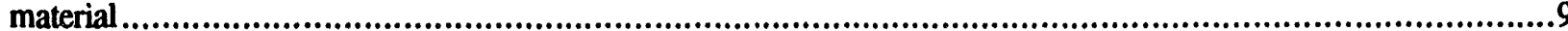

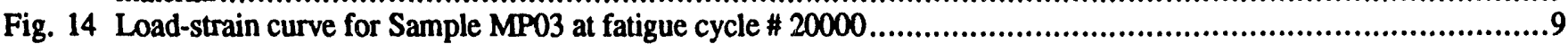

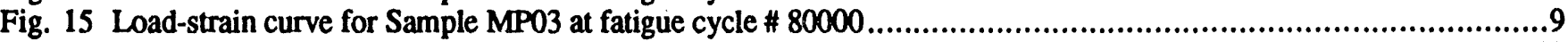

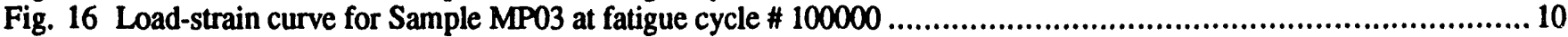

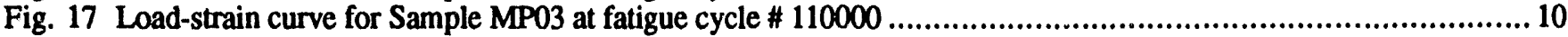

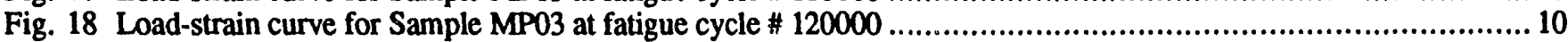

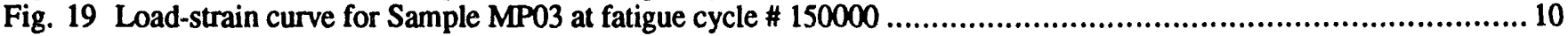

Fig. 20 Variation of tensile load and coercivity with expended fatigue life for Sample EPI 1 under fatigue test with strain amplitude of 0.003

Fig. 21 Variation of remanence and hysteresis loss with expended fatigue life for Sample EPI 1 under fatigue test with

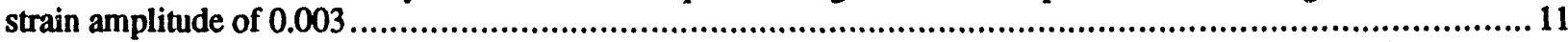

Fig. 22 Variation of tensile load and coercivity with expended fatigue life for Sample EP09 under fatigue test with strain amplitude of 0.003

Fig. 23 Variation of remanence and maximum permeability with expended fatigue life for Sample EP09 under fatigue test with strain amplitude of 0.003

Fig. 24 Variation of tensile load and coercivity with expended fatigue life for Sample EP10 under fatigue test with strain amplitude of 0.003

Fig. 25 Variation of remanence and maximum permeability with expended fatigue life for Sample EP10 under fatigue

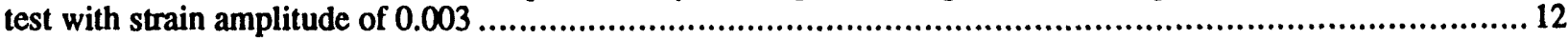

Fig. 26 Barkhausen signal as a function of fatigue life at penetration depth of $0.2 \mathrm{~mm}$ and $0.07 \mathrm{~mm}$ for Sample EP06........ 13

Fig. 27 Barkhausen signal as a function of fatigue life at penetration depth of $0.2 \mathrm{~mm}$ for Sample EP06, comparing original data with the data processed by DFT ................................................................................ 13

Fig. 28 Barkhausen signal as a function of fatigue life at penetration depth of $0.07 \mathrm{~mm}$ for Sample EP06, comparing

Fig. 29 Barkhausen signal as a function of fatigue life at penetration depth of $0.2 \mathrm{~mm}$ for Sample EP07, comparing 13

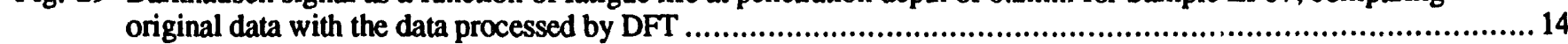

Fig. 30 Barkhausen signal as a function of fatigue life at penetration depth of $0.07 \mathrm{~mm}$ for Sample EP07, comparing original data with the data processed by DFT .................................................................................. 14

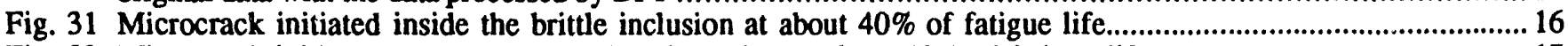

Fig. 32 Microcrack initiated at the edge of brittle inclusion at about $40 \%$ of fatigue life.............................................. 17

Fig. 33 Growth of microcrack due to fatigue at about $60 \%$ of fatigue life ..................................................... 18

Fig. 34 Growth of microcrack due to fatigue at about $70 \%$ of fatigue life .......................................................... 19

Fig. 35 Distribution of inclusions inside A533B steel (at 65X magnification) ...........................................................20

Fig. 36 Line distribution of inclusions inside A533B steel (at 65X magnification).................................................220

Fig. 37 Distribution of inclusions on grain boundary inside A533B steel (at 65X magnification)................................21

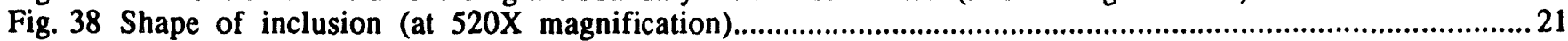

Fig. 39 Variation of coercivity at two different locations with expended fatigue life for Sample MP10 under fatigue test with stress amplitude of $272 \mathrm{MPa}$. 
Fig. 40 Variation of remanence and hysteresis loss with expended fatigue life for Sample MP10 under fatigue test with stress amplitude of $272 \mathrm{MPa}$

Fig. 41 Variation of compressive and tensile strain with expended fatigue life for Sample MP12 under fatigue test with stress amplitude of $272 \mathrm{MPa}$

Fig. 42 Variation of tensile strain with expended fatigue life for Sample MP13 under fatigue test with stress amplitude of $243 \mathrm{MPa}$.

Fig. 43 Variation of coercivity and remanence with expended fatigue life for Sample MP15 under fatigue test with stress amplitude of $284 \mathrm{MPa}$.

Fig. 44 Variation of coercivity and remanence with expended fatigue life for Sample MP16 under fatigue test with stress amplitude of $284 \mathrm{MPa}$.

Fig. 45 Variation of coercivity and remanence with expended fatigue life for Sample PRE5 under fatigue test with stress

Fig. 46 Variation of hysteresis loss and tensile load with expended fatigue life for Sample PRES under fatigue test with

Fig. 47 Variation of coercivity and hysteresis loss with expended fatigue life for Sample PREl under faigue test with stress amplitude of 0.003 . The specimen was prestrained with residue strain of 0.021 ..................................25

Fig. 48 Variation of coercivity and remanence with expended fatigue life for Sample PRE1 under fatigue test with stress amplitude of 0.003 . The specimen was prestrained with residue strain of 0.021 


\section{Overview}

\subsection{Objective}

The objective of the research undertaken in this project was to investigate the application of new advanced magnetic inspection techniques to the detection of fatigue damage in steels used in the nuclear industry, and to demonstrate the possibility of in situ evaluation of the changes in the mechanical condition of the steel using magnetic methods.

\subsection{Potential Benefits}

If methods can be identified which can accurately determine accumulated fatigue damage in a material, and thereby give an estimation of the remaining fatigue life, the impact on the cost of maintenance and safe operation of nuclear power plants will be enormous. Currently destructive tests are used on specimens which are removed from components in service. This clearly has detrimental effects on the components, even when repairs are made, and the situation is all the more unsatisfactory when the sample which was removed proved to be in good condition for continued service. The cost of these destructive tests is also not to be underestimated. Therefore the present work was designed to identify inagnetic measurements which could be used to assess the condition of steel components without removal of any material from the component.

\subsection{Summary of Main Tasks}

In order to show a correlaiion between the fatigue damage accumulation and the variation of magnetic properties, the project consisted of the following tasks: preparing the A533B fatigue samples (tensile specimens); performing both low cycle and high cycle fatigue; taking magnetic hysteresis measurements at predetermined fatigue cycle intervals; performing magnetic Barkhausen measurements and comparing the results with hysteresis measurements; undertaking metallographic investigation to correlate fatigue damage with the magnetic measurements; performing fatigue tests on prestrained samples to investigate the influence of the initial mechanical state on fatigue life and magnetic properties.

\subsection{Approach}

Ine research extended the techniques developed at the Center for NDE at Iowa State University to the problem of inspection of fatigue damage in steel components of nuclear systems. Since the ultimate goal is to inspect the nuclear power generating system for assessment of reliability or remaining service life, the laboratory measurements provided basic magnetic property data under well controlled fatigue conditions.

Measurements of magnetic properties of A533B steel were made throughout the whole fatigue life. These laboratory tests were designed to investigate whether the magnetic properties changed as a result of fatigue.

The work was divided broadly into three parts:

(1) Fatigue damage studies

(2) Comparison of different magnetic measurement techniques

(3) Correlation of the variation of magnetic properties with fatigue damage

The first part involved sample preparation, various fatigue tests and measurements of mechanical and magnetic properties. Forty tensile specimens were machined along with two specially designed inspection heads which were cont sured to the shape of the test samples. Tensile tests and hardness tests were performed, followed by intensive fatigue testing under different conditions both in the low cycle and the high cycle fatigue regions. Magnetic properties were measured by a portable magnetic inspection system, the Magnescope [1].

The second part involved the identification of suitable magnetic NDE techniques for evaluation of remaining lifetime. Both magnetic hysteresis measurement techniques and Barkhausen measurement techniques were reported as good candidates for detecting changes in the mechanical 
condition of these steels. In this project, we have assessed the relative advantages of Barkhausen systems (e.g., "Stresscan" [2]) and hysteresis systems (e.g., Magnescope), in terms of our ability to interpret results, the repeatability of results and the ease of measurement.

The third part of the investigation was to interpret the variation of magnetic properties in terms of fatigue damage caused by cyclic loading.

Although the actual mechanism by which fatigue influenced magnetic properties remains problematic, the test results obtained and the models developed during this project have led to a better understanding of this problem, and should lead to the development of viable magnetic NDE technology.

\subsection{Personnel}

During the past two years, two professors, one postdoctoral fellow and one graduate student were involved in the project. Dr. D.C. Jiles, a senior physicist at Ames Laboratory and a Full Professor of Materials Science and Engineering and Electrical Engineering and Dr. S.B. Biner, an Associate Metallurgist at Ames Laboratory and Assistant Professor of Materials Science and Engineering were the principal investigators of this project. Dr. Govindaraju, a metallurgist with expertise in material characterization, was hired for the postdoctoral position. A graduate student, Zhao-Jun Chen, working for a Ph.D. in Electrical Engineering, was hired as research assistant and performed laboratory fatigue tests and magnetic measurements. Also Dr. J. Kameda, a metallurgist at Ames Laboratory, was involved in metallographic studies and interpreting the test results. The combination of the people with expertise in different areas made a team with broad experience which carried this project to a successful conclusion. 


\section{Background}

\subsection{Magnetic Inspection Methods}

Magnetic NDE methods are currently undergoing an unprecedented expansion. There are two main reasons: ferritic steels are widely used as a constructional material and their magnetic properties are highly sensitive to their mechanical state. Investigations in magnetic NDE technologies have been reviewed recently $[3,4]$ and this showed that the techniques such as the Barkhausen effect [5,6,7], magnetoacoustic emission $[8,9,10]$ and magnetic hysteresis $[11,12,13]$ have been used to evaluate the mechanical condition of materials including stress effects [14], plastic deformation [15], creep damage [16] and fatigue damage [17].

As a result of progress in these techniques, and in view of the accumulation of fatigue in components of nuclear power systems, it was expected that the utilization of our knowledge of magnetism could be advantageously turned towards solving at least a significant part of the problem of monitoring nuclear power system for fatigue damage.

It is well-known that fatigue failures are a very serious problem due to the insidious nature of the failure. Fatigue damage studies have shown that magnetic hysteresis measurements can be used for continuous monitoring of fatigue damage in steels and that the coercivity and remanence appear to be particularly sensitive to remaining life in the last $10 \%$ of fatigue life. This sensitivity, which is manifested as sudden changes in magnetic parameters such as coercivity and remanence, occurs before any visible signs of impending failure arise in the materials [Appendix Al.4].

\subsection{Sensitivity of Magnetic Properties to Fatigue}

Recently magnetic techniques for inspection of the mechanical properties of ferritic steels have developed very rapidly. This has been shown through the work of groups in the UK (Harwell Laboratory), Canada (Queen's University), Germany (Fraunhofer Institute, Universitat des Saarlandes) and United States (Center for NDE, Iowa State University). Not only are techniques such as Barkhausen effect, magnetoacoustic emission and magnetic hysteresis viable, they are also highly sensitive to changes in mechanical condition and represent methods with a high potential for successful inilustrial exploitation.

The results presented here are consistent with earlier results $[18,19]$ which showed that the magnetic inspection parameters are strongly influenced by the mechanical condition of the material. It is also shown that under certain conditions, quantitative estimation of fatigue damage is possible.

In conjunction with the current problem of fatigue in components of nuclear power systems, it is appropriate that magnetic inspection methods be given high priority in a search for reliable methods for assessment of remaining lifetime of nuclear pressure vessel steel. The present research project has investigated two of these methods, Barkhausen effect and magnetic hysteresis measurements, with the objective of understanding the effects of fatigue on the magnetic properties. This project has assessed the suitability of these methods for determining the fatigue life of nuclear pressure vessel, and has explored design modifications of the existing magnetic measuring equipment to meet the needs of or-site inspection. 


\section{Fatigue Studies on Materials Under Laboratory Conditions}

\subsection{Fatigue Testing}

In order to correlate fatigue damage accumulation with magnetic properties and the influence of initial state of the material on fatigue behavior, fatigue studies were conducted on both as-received and prestrained specimens.

The as-received specimens were tested in both high-cycle and low-cycle fatigue regimes. The S$N, \varepsilon-N$ curves were generated and stress-strain loops also were recorded for the material under appropriate fatigue regimes. The fatigue tests were conducted by using a computer controlled, 20KIPS, servo hydraulic MTS testing system at Ames Laboratory. The unit was interfaced with data acquisition and control cards via a personal computer. The software was designed to carry out fatigue tests under load or strain amplitude control as well as to record load and strain data. Since the data acquisition and control were achieved simultaneously, the test variables (load or strain amplitudes) were always kept constant, using the recorded test data to set the control signals.

The magnetic signature was found to be very sensitive to external testing conditions. A fatigue test stop at a down-stroke or an up-stroke could make a significant difference in the measured magnetic properties. To maintain the comparability of results, the MT'S control software was modified to start and stop the tests with completion of a full fatigue cycle. For both low cycle and high cycle fatigue tests, load or strain were sinusoidal and the frequency was between 1 and $4 \mathrm{~Hz}$.
The magnetic properties were measured by interrupting the fatigue test at predetermined intervals in the fatigue lifetime. (The intervals were chosen at both linear and logarithmic increments). The Magnescope was used to measure hysteresis parameters while a commercially available instrument Stresscan was used to detect Barkhausen amplitude. These magnetic parameters were recorded for correlation with the expended fatigue life. Some specimens were subsequently metallographically examined. Fatigue damage and nucleation of fatigue cracks were investigated using both scanning electron microscopy(SEM) and optical microscopy.

\subsection{Fatigue Analysis}

The fatigue life has been defined as the number of cycles needed for either complete failure of the specimen in load-controlled fatigue tests or over 50 percent decrease in load in the strain-controlled fatigue tests. The fatigue life was used to interpret the resistance of the material to stress and load cycle.

During the tests, the load, and strain data were continuously recorded. Due to the formation of stable microcracks at the later stages of fatigue life, a decrease in load level in strain-controlled fatigue tests and an increase in the strain data for loadcontrolled fatigue tests usually occurred as a result of loss of stiffness. This information was recorded to compare with magnetic measurements in order to identify the source of variation in magnetic parameters, and thus to study the sensitivity of magnetic NDE technology for fatigue damage evaluation. 


\section{Results}

The results of this study may be summarized as follows: significant changes in magnetic properties were observed during fatigue tests both under strain control and load control. Magnetic hysteresis parameters showed a sudden change in the last $10-20 \%$ of fatigue life in strain-controlled tests, however, their changes were continuous in load-controlled tests. Barkhausen effect measurements showed that the Barkhausen amplitude changed continuously in both cases. Further detailed studies showed that during the load-controlled high cycle fatigue, the magnetic hysteresis parameters, such as coercivity, remanence and hysteresis loss changed linearly with the logarithm of the number of fatigue cycles. Therefore a quantitative relationship between coercivity and fatigue life can be given based on two empirical coefficients. Also metallographic studies showed that inclusions in A533B steel could have an important effect in fatigue crack initiation.

\subsection{Milestone Progress Review}

1. Two pieces of A533B steel with dimensions of $10 \mathrm{~cm} \times 10 \mathrm{~cm} \times 30 \mathrm{~cm}$ and $10 \mathrm{~cm} \times 10 \mathrm{~cm} \times$ $20 \mathrm{~cm}$ were received from Oak Ridge National Laboratory. These pieces were broken halves from the Heavy Section Steel Technology (HSST) shallow-crack program. The larger piece was from the HSST-CE plate material and the smaller piece was from HSST plate 13B. Characterization information on these two heats of material was also received at the same time. 40 smooth fatigue specimens in "hour glass" shape with $6 \mathrm{~mm}$ diameter and 51 $\mathrm{mm}$ gauge length, parallel to the rolling direction were machined from the material.

2. Tensile tests to determine the strength characteristics of the steel were conducted. The material exhibited $0.2 \%$ yield strength in the range of 356 to $381 \mathrm{MPa}$ with ultimate tensile strength of 517 to $555 \mathrm{MPa}$. Hardness tests were also performed and the average Rockwell hardness was about $88 R_{B}$.

3. Two inspection heads were carefully designed to have a close fit to the geometry of the test samples. Each inspection head contained a flux coil for measuring magnetic induction, a Hall probe for measuring magnetic field, and a power coil.

4. Initial fatigue tests were conducted focusing on low cycle fatigue with strain amplitude of 0.003 . The variation in magnetic properties during the tests are shown in the results section, together with a discussion of the underlying mechanism behind the observed changes. Fatigue tests at other strain levels were also performed. The results of magnetic measurement were similar.

5. Barkhausen measurements were performed using a portable commercial Barkhausen effect instrument Stresscan 500C. Barkhausen signals were compared with hysteresis parameters.

6. Metallurgical studies were performed by observing the surface condition of the fatigue damaged samples under SEM. Optical microscopy pictures were also taken to evaluate the morphology of the material.

7. Fatigue tests under load-controlled condition at different load levels were performed. The magnetic parameters were measured using the Magnescope and are shown in subsequent sections.

8. Prestrain fatigue tests were performed and test results were compared with the results of the unprestrained tests.

9. Two interim reports have been submitted during this research program. The first was submitted after one year (November, 1992) and the other one was after a year and a half (May, 1993).

\subsection{Mechanical Property Measurements}

Tensile tests were performed on two specimens cut from HSST-CE plate material in order to determine both yield strength and ultimate tensile strength of the test material. The results are shown in Figs. 1-2. From the data, the following properties were determined by averaging the results on the two specimens. 
Results

Yield strength $=368 \mathrm{MPa}$

$0.2 \%$ yield strength $=369 \mathrm{MPa}$

Ultimate tensile strength $=536 \mathrm{MPa}$

Rockwell hardness tests were performed on broken pieces of these two samples. The average Rockwell hardness was $88 \mathrm{R}_{\mathrm{B}}$.

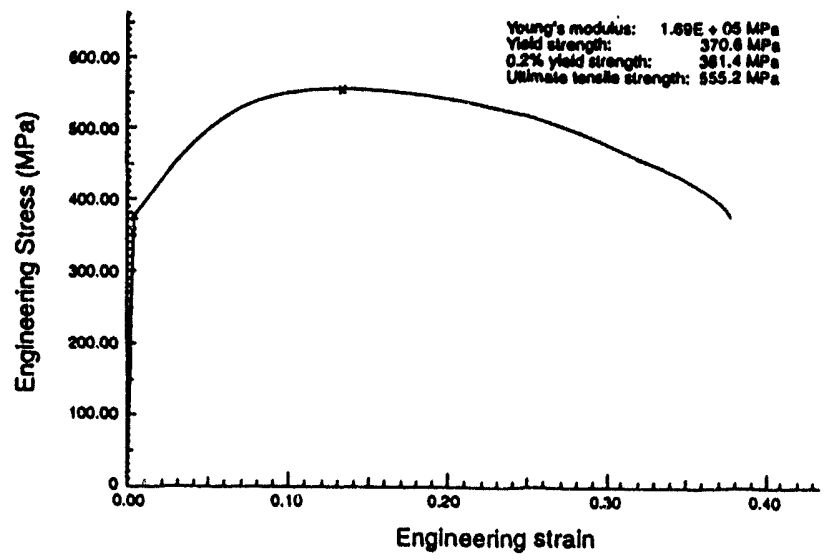

Fig. 1 Stress-strain curve for 1st specimen of A533B steel.

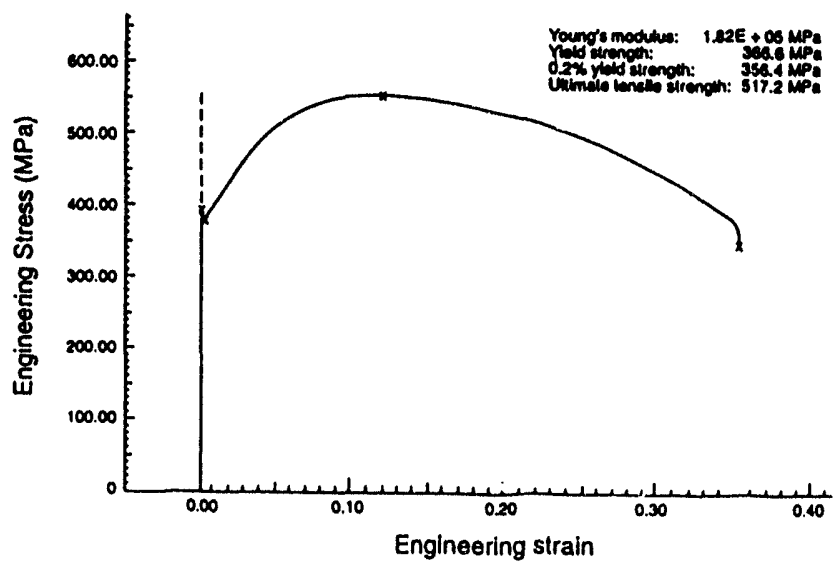

Fig. 2 Stress-strain curve for 2 nd specimen of A533B steel.

\subsection{Strain-Controlled Low Cycle Fatigue Test}

Fatigue tests were initially performed at different strain levels from 0.0015 to 0.007 . Mechanical hysteresis loops were obtained after a predetermined number of fatigue cycles. These loops could be used to determine whether the fatigue test was conducted under low cycle fatigue conditions or under high cycle fatigue conditions, and could also be used to find the onset of the formation of macrocracks on observing the distortion of the loop. A typical S-N curve is shown in Fig. 4 and a series mechanical hysteresis loops for Sample EP05 with strain amplitude of 0.003 are shown in Figs. 4 - 10. From Fig. 4, it can be seen that under low cycle fatigue, mechanical hysteresis occurred very early in the fatigue life. From Fig. 9 it can be seen that a macrocrack began to propagate before 16,000 stress cycles. Similar fatigue test results at smaller strain amplitude of 0.0018 for Sample MP03 are shown in Figs. 11 - 19. It can be seen that in Sample MP03, under high cycle fatigue testing, the macrocrack formed before 110,000 stress cycles.

FATIGUE TEST:LOAD-CYCLE SAMPLE:EP05, STRAIN: |0.003|

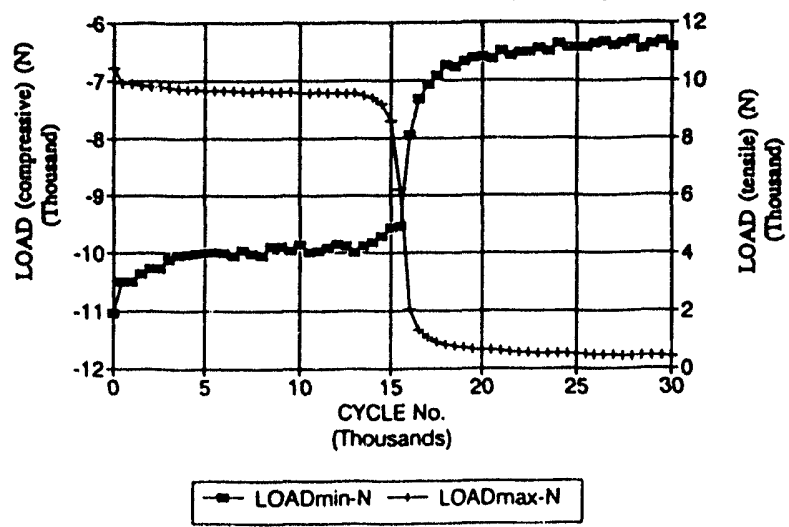

Fig. 3 Variation of tensile and compressive load with expended fatigue life for Sample EP05 with fatigue strain amplitude of 0.003 . 
Results

FATIGUE TEST: LOAD-STRAIN SAMPLE:EP05; STRIN $=|0.003|$

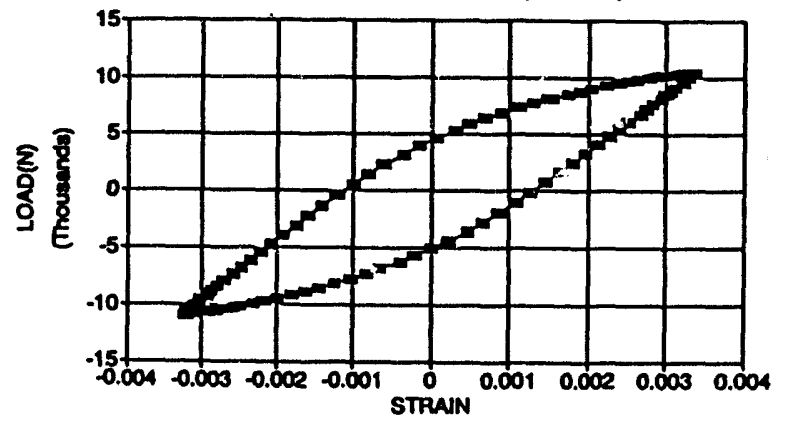

$-N=5$

Fig. 4 Load-strain curve for Sample EP05 at fatigue cycle \# 5 .

FATIGUE TEST: LOAD-STRAIN SAMPLE:EP05; STRIN $=|0.003|$

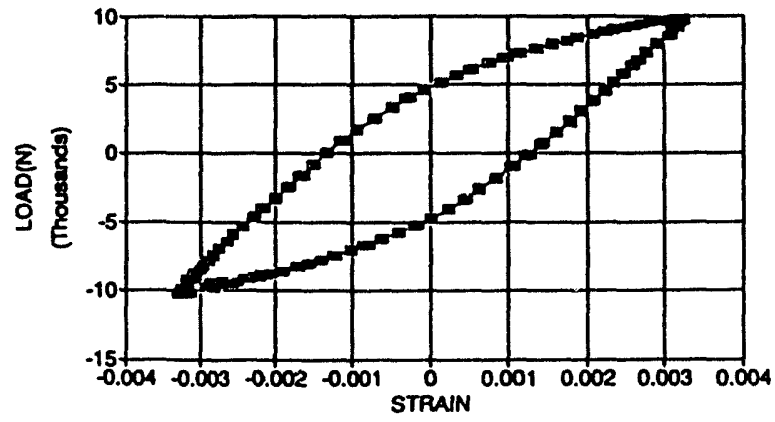

$-N=2000$

Fig. 5 Load-strain curve for Sample EP05 at fatigue cycle of 2000 .

FATIGUE TEST: LOAD-STRAIN SAMPLE:EPD5; STRIN $=|0.003|$

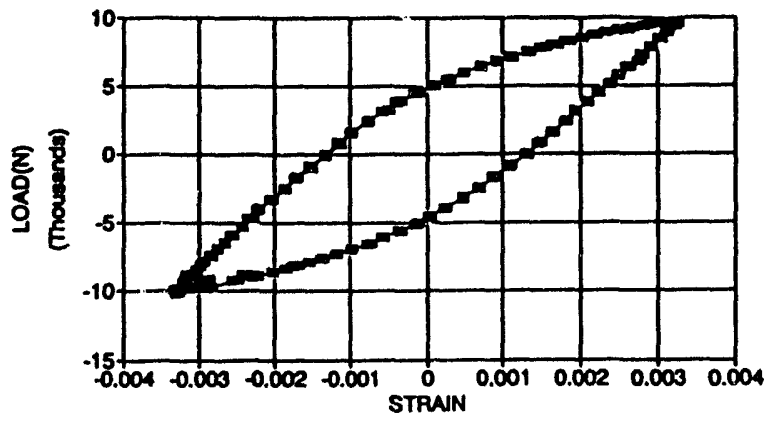

$\rightarrow-N=4000$

Fig. 6 Load-strain curve for Sample EP05 at fatigue cycle \# 4000 .

FATIGUE TEST: LOAD-STRAIN SAMPLE:EP05; $S T R I N=|0.003|$

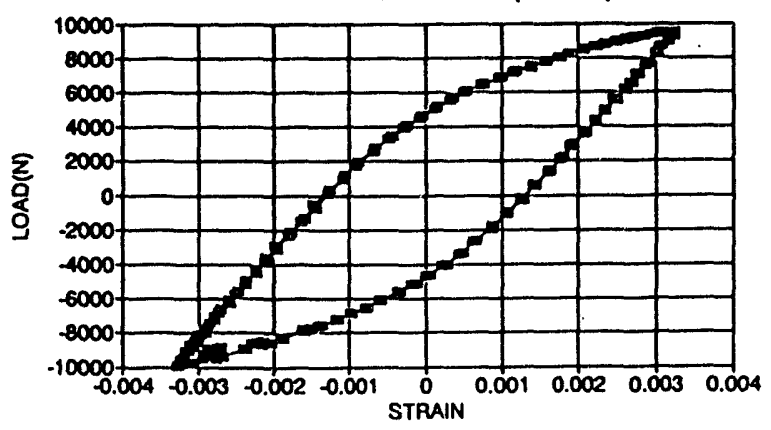

$\rightarrow-N=12000$
Fig. 7 Load-strain curve for Sample EP05 at fatigue cycle \# 12000 . 
FATIGUE TEST: LOAD-STRAIN SAMPLE:EP05; STRIN $=|0.003|$

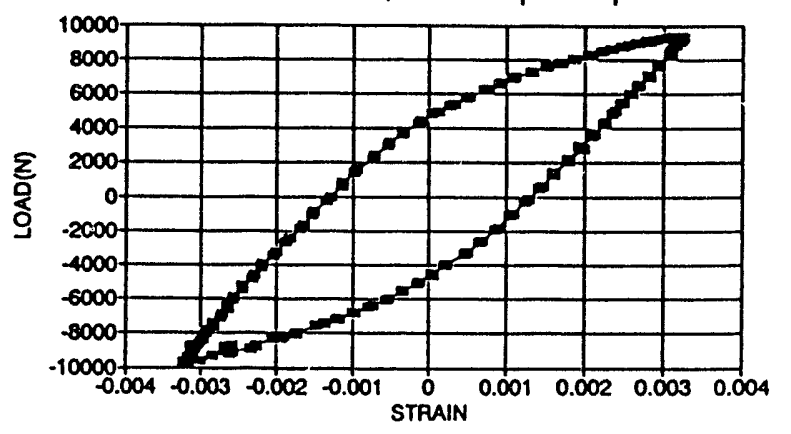

$-\infty-N=14000$
FATIGUE TEST: LOAD-STRAIN SAMPLE:EP05; STRIN $=|0.003|$

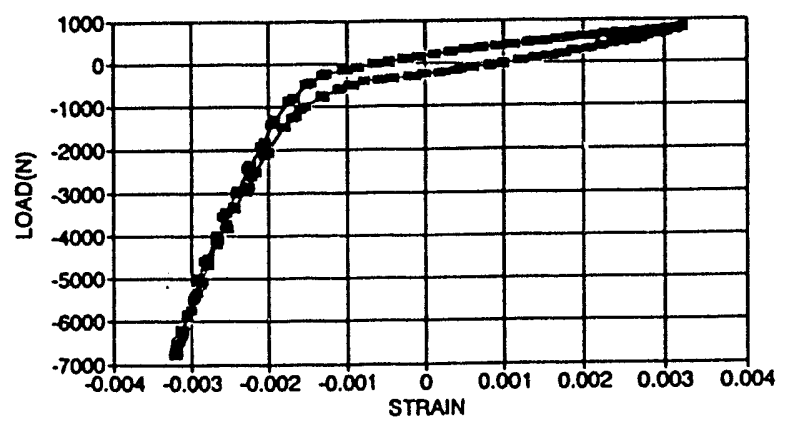

$-N=18000$

Fig. 10 Load-strain curve for Sample EP05 at fatigue cycle \# 18000 .

FATIGUE TEST: LOAD-CYCLE SAMPLE:MP03; STRAIN $=|0.0018|$

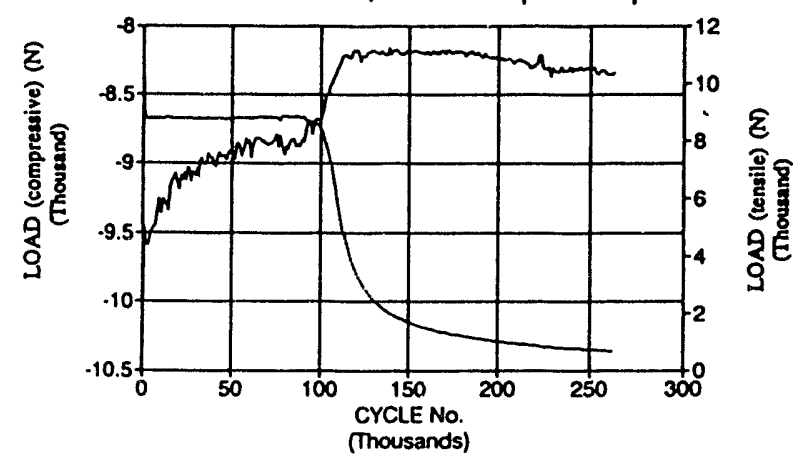

- LOADmin.N - LOADmax-N
Fig. 9 Load-strain curve for Sample EP05 at fatigue cycle \# 16000 .
Fig. 11 Variation of tensile and compressive load with expended fatigue life for Sample MP03 with fatigue strain amplitude of 0.0018 . 
Results

FATIGUE TEST: LOAD-STRAIN LOOP SAMPLE: HFMP03, STRAIN $=|0.0018|$

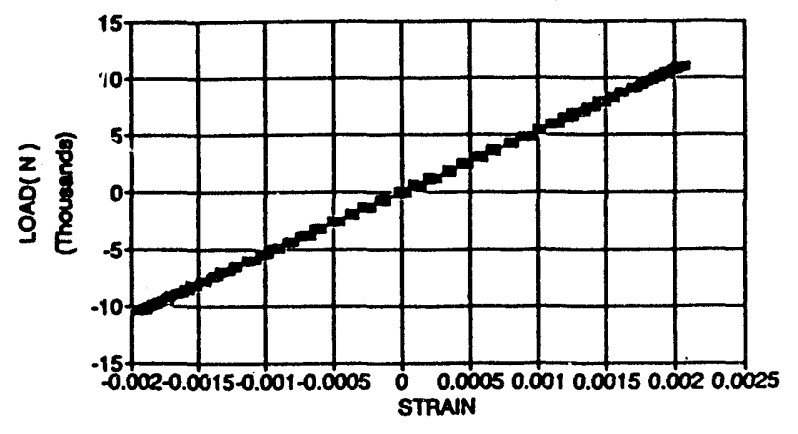

$-N=5$

Fig. 12 Load-strain curve for Sample MP03 at fatigue cycle \# 5 .

FATIGUE TEST: LOAD-STRAIN LOOP SAMPLE: HFMP03, STRAIN $=|0.0018|$

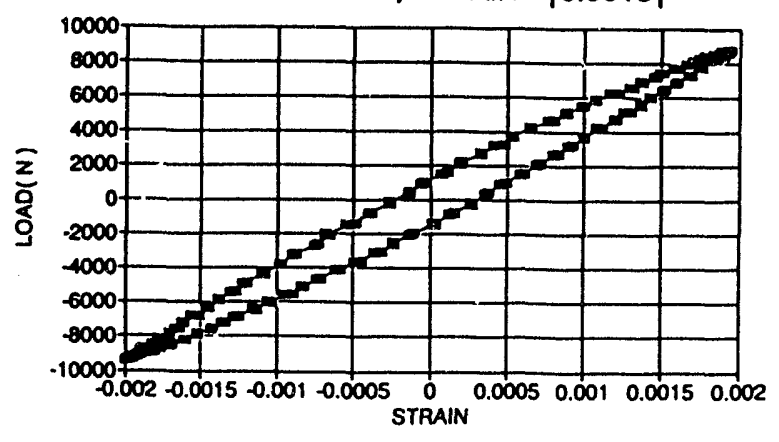

$-N=10000$

Fig. 13 Load-strain curve for Sample MP03 at fatigue cycle \# 10000 , area within loop indicating micro-yielding of material.
FATIGUUE TEST: LOAD-STRAIN LOOP SAMPLE: HFMP03, STRAIN $=|0.0018|$

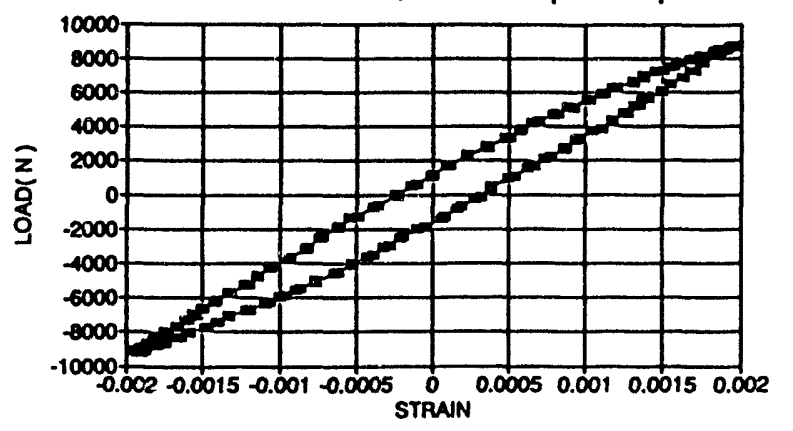

$-N=20000$

Fig. 14 Load-strain curve for Sample MP03 at fatigue cycle \#20000.

\section{FATIGUE TEST: LOAD-STRAIN LOOP SAMPLE: HFMP03, STRAIN $=|0.0018|$}

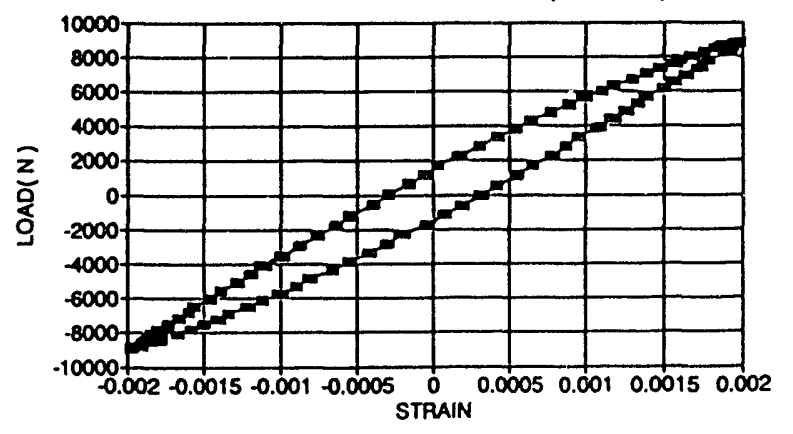

$-N=80000$

Fig. 15 Load-strain curve for Sample MP03 at fatigue cycle \#80000. 
FATIGUE TEST: LOAD-STRAIN LOOP SAMPLE: HFMP03, STRAIN $=|0.0018|$

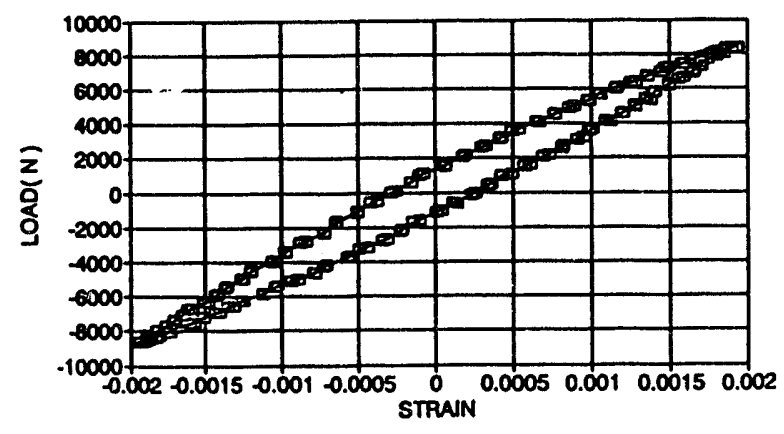

$-E-N=100000$
FATIGUE TEST: LOAD-STRAIN LOOP SAMPLE: HFMP03, STRAIN $=|0.0018|$

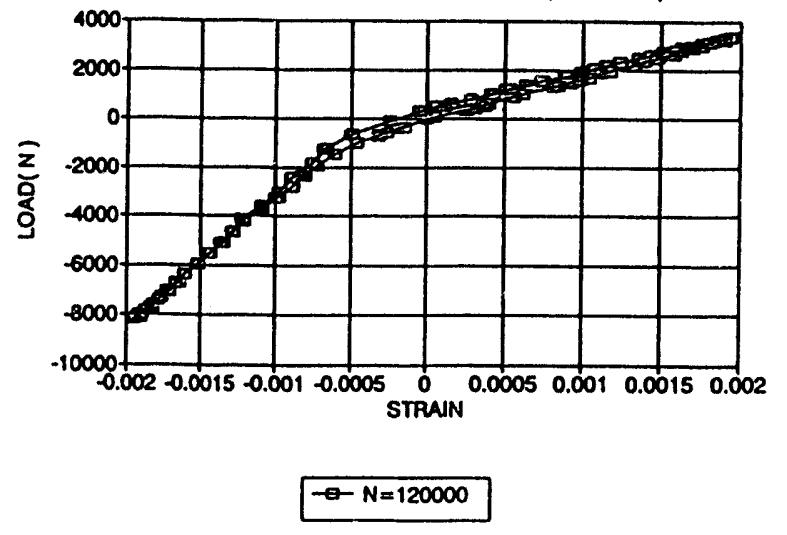

Fig. 18 Load-strain curve for Sample MP03 at fatigue cycle \#120000.

Fig. 16 Load-strain curve for Sample MP03 at fatigue cycle \# 100000 .

\section{FATIGUE TEST: LOAD-STRAIN LOOP SAMPLE: HFMP03, STRAIN $=|0.0018|$}

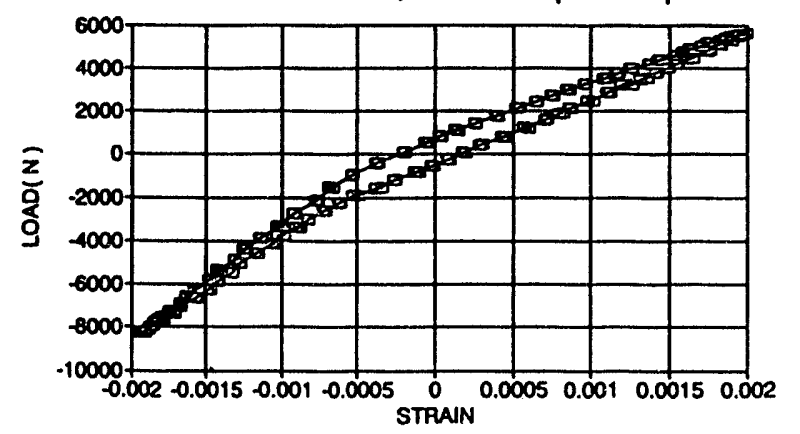

$\square-N=110000$

Fig. 17 Load-strain curve for Sample MP03 at fatigue cycle \# 110000 .

NUREG/GR-0013

\section{FATIGUE TEST: LOAD-STRAIN LOOP SAMPLE: HFMP03, STRAIN $=|0.0018|$}

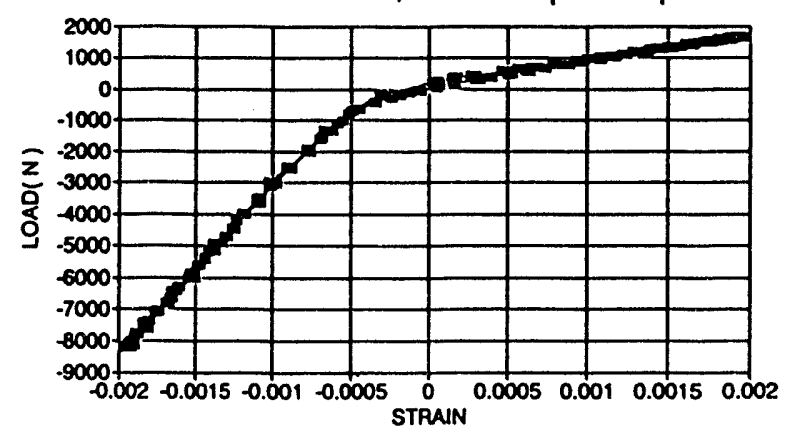

$-N=150000$

Fig. 19 Load-strain curve for Sample MP03 at fatigue cycle \# 150000 .

The strain amplitude of 0.003 was chosen as a convenient condition for a systematic study of the effects of low cycle fatigue on magnetic properties was evident from the mechanical hysteresis curve (Fig. 4), and the fatigue life was typically around 15,000 cycles before failure. of these steels. At this strain level, plastic strain 
Magnetic hysteresis parameters were measured using the Magnescope at predetermined intervals throughout the whole fatigue life. Test results for specimen EP11 with strain amplitude of 0.003 are shown in Figs. 20 - 21. Similar results for a different specimens EP09 and EP10 are shown in Figs. $22-25$. It can be seen that the coercivity decreased moderately in the early stage of fatigue life, as a result of fatigue softening. Then the coercivity and other magnetic properties remained quite stable over most of the fatigue life. This stable region amounted to $80-90 \%$ of the lifetime. Finally in the last $10-20 \%$ of fatigue life, the magnetic properties changed dramatically. According to the load profile, the final stage which corresponded to the formation of a macrocrack, was accompanied by a decreasing load that was needed to obtain the constant strain amplitude. It was expected that the bulk microstructure would be stable after the initial fatigue softening. The invariance of the magnetic parameters throughout the intermediate range of fatigue life was consistent with such an interpretation.

FATIGUE TEST, SAMPLE: EP11 STRAIN: $|0.003|$

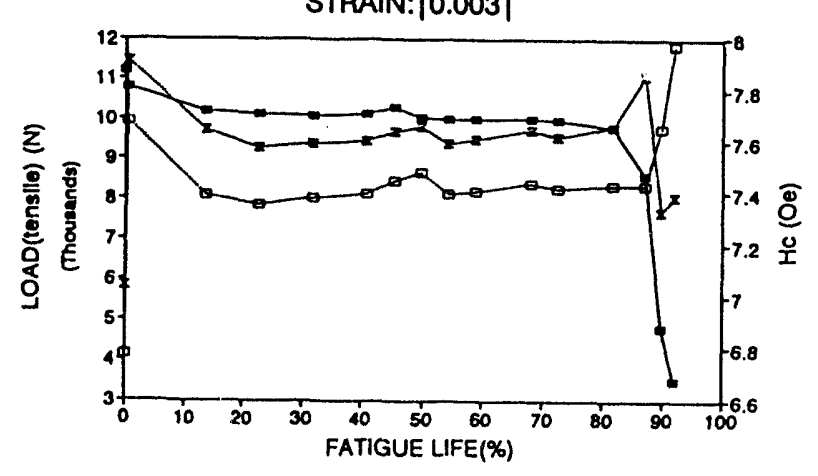

Fig. 20 Variation of tensile load and coercivity with expended fatigue life for Sample EPI 1 under fatigue test with strain amplitude of 0.003 .

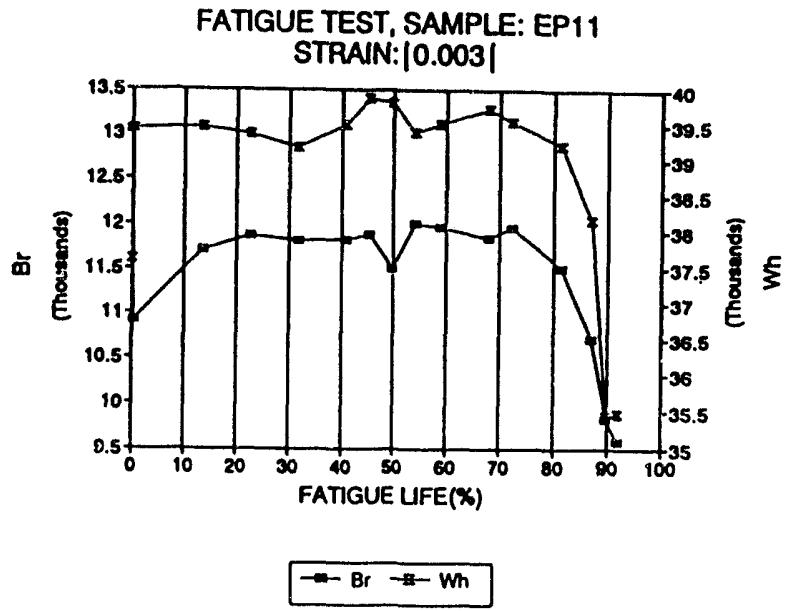

Fig. 21 Variation of remanence and hysteresis loss with expended fatigue life for Sample EPI 1 under fatigue test with strain amplitude of $\mathbf{0 . 0 0 3}$.

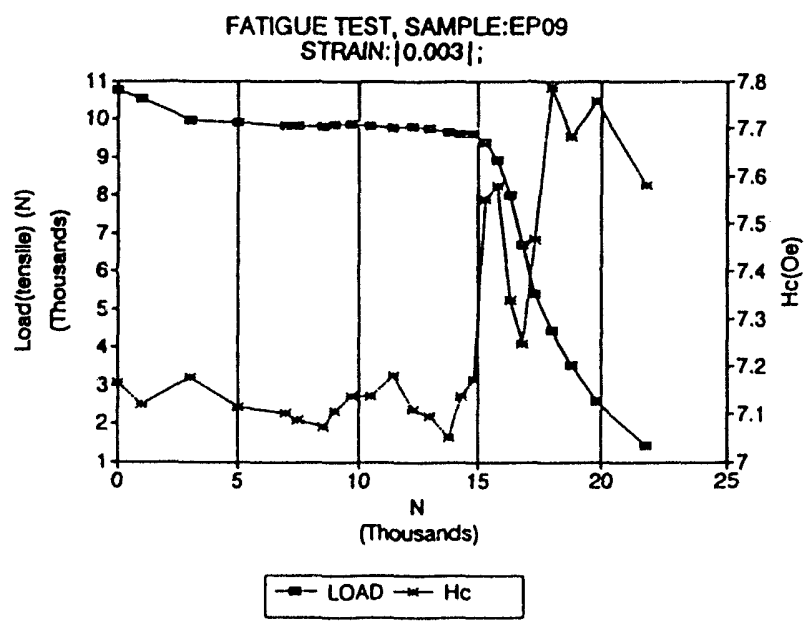

Fig. 22 Variation of tensile load and coercivity with expended fatigue life for Sample EP09 under fatigue test with strain amplitude of $\mathbf{0 . 0 0 3}$. 


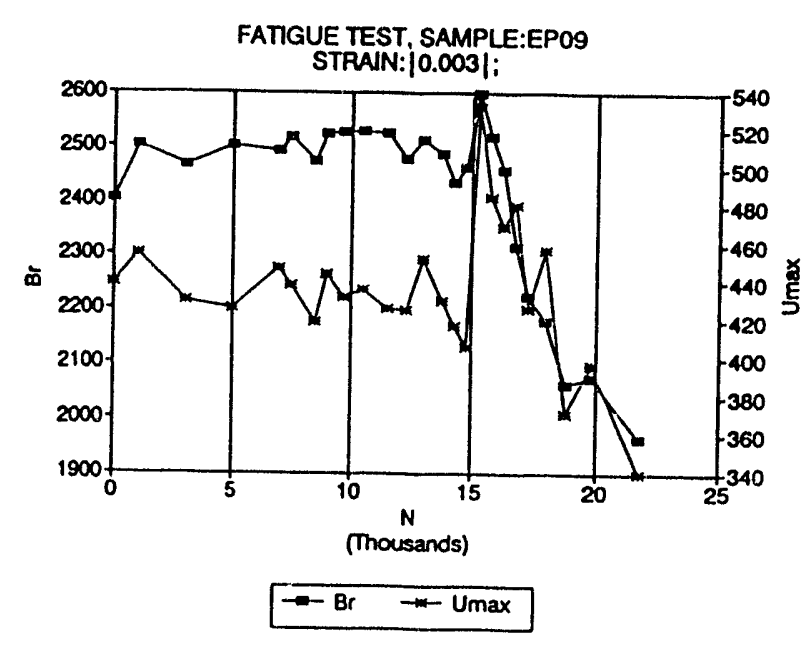

Fig. 23 Variation of remanence and maximum permeability with expended fatigue life for Sample EP09 under fatigue test with strain amplitude of $\mathbf{0 . 0 0 3}$.

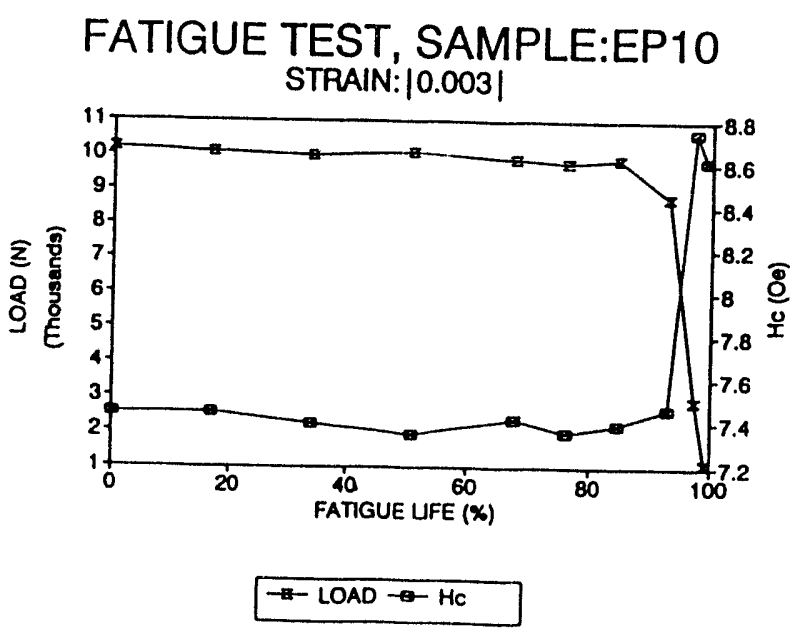

Fig. 24 Variation of tensile load and coercivity with expended fatigue life for Sample EP10 under fatigue test with strain amplitude of $\mathbf{0 . 0 0 3}$.

\section{FATIGUE TEST, SAMPLE:EP10} STRAIN:|0.003|

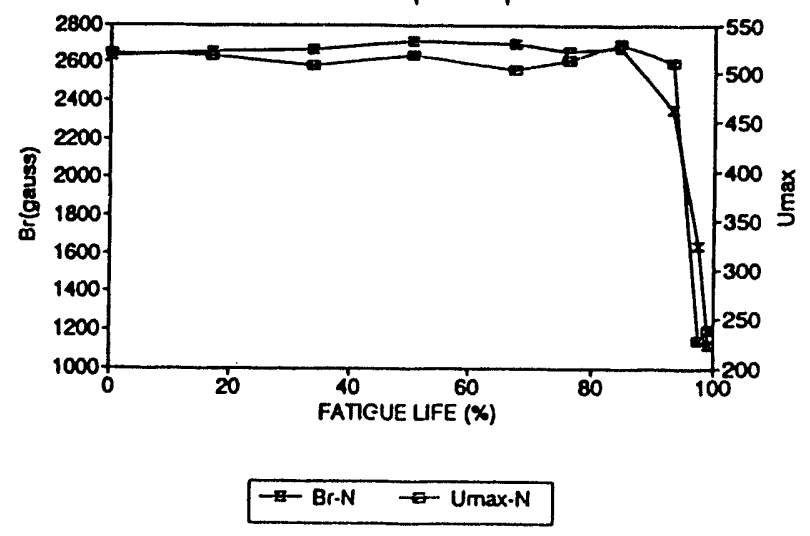

Fig. 25 Variation of remanence and maximum permeability with expended fatigue life for Sample EP10 under fatigue test with strain amplitude of $\mathbf{0 . 0 0 3}$.

\subsection{Barkhausen Effect Measurements}

Barkhausen effect measurement were performed to investigate the dependence of Barkhausen signal on the expended fatigue life with the objective of detecting fatigue damage even before the stage of macrocrack propagation.

The rational for this investigation was that fatigue failure primarily begins on the surface. The formation of extrusion and intrusion due to slip at the surface of a specimen can lead to high surface stresses which nucleate a microscopic crack, which will later form a macroscopic crack and propagate through the sample. If this is the case, the most important property to monitor is the surface stress. By their nature, Barkhausen emissions, which have an effective frequency range typically from $20-300 \mathrm{kHz}$, have a short penetration range in steels. The corresponding penetration range is typically $0.05-0.2 \mathrm{~mm}$, which means that the Barkhausen emissions really give information only about the surface layer of the material. Therefore, this technique could be viable in determining the process of fatigue damage, and 
consequently Barkhausen effect measurements seen to be ideally suited for these type of surface studies.

The Stresscan was used to detect the Barkhausen amplitude during the strain-controlled fatigue test with strain amplitude of 0.003 . Test results are shown in Figs. 26 - 30. It can be seen that the Barkhausen amplitude changed continuously during the whole fatigue life. These results indicated that the whole fatigue lifetime can be divided into three stages based on the changes in the Barkhausen signal.

\section{FATIGUE TEST: M.P. MEASUREMENT} SAMPLE: LFEP06; STRAIN:|0.003|

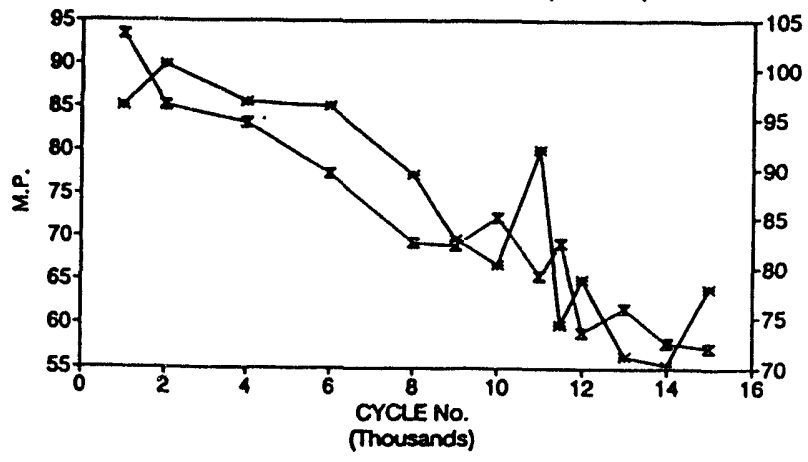

$\rightarrow$ RAW DATA:d $=0.2 \mathrm{~mm} \rightarrow-$ RAW DATA:d $=0.07 \mathrm{~mm}$

Fig. 26 Barkhausen signal as a function of fatigue life at penetration depth of $0.2 \mathrm{~mm}$ and $0.07 \mathrm{~mm}$ for Sample EP06.
Fatigue test: M.P. Measurement SAMPLE EP06; STRAIN: |0.003|

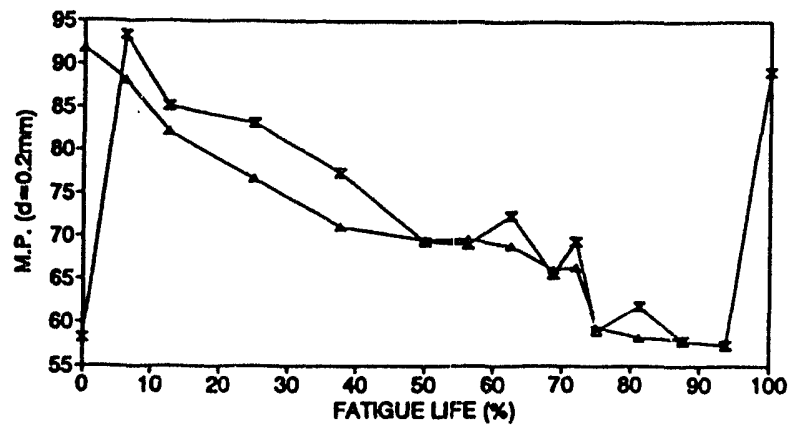

$\rightarrow$ OBSERVED DATA $\sim$ PROCESSED DATA

Fig. 27 Barkhausen signal as a function of fatigue life at penetration depth of $0.2 \mathrm{~mm}$ for Sample EP06, comparing original data with the data processed by DFT.

Fatigue test: M.P. Measurement SAMPLE EP06; STRAIN: $|0.003|$

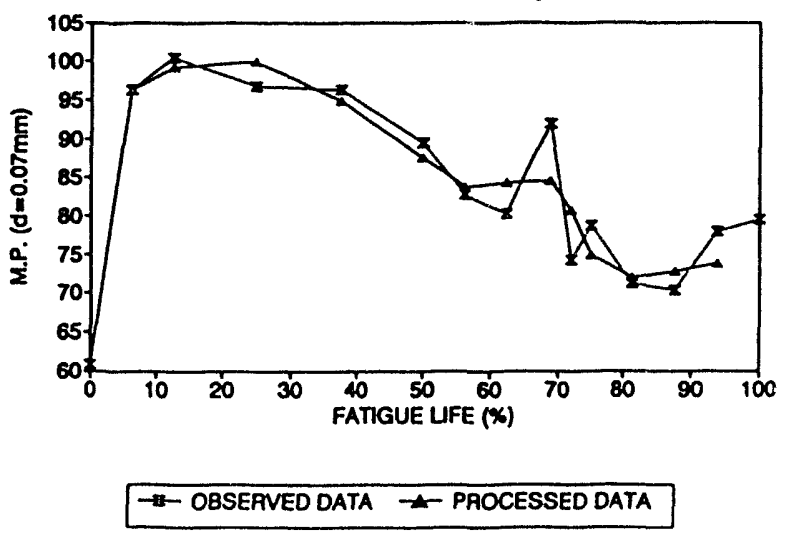

Fig. 28 Barkhausen signal as a function of fatigue life at penetration depth of $0.07 \mathrm{~mm}$ for Sample EP06, comparing original data with the data processed by DFT. 
Fatigue test: M.P. Measurement SAMPLE EP07; STRAIN:|0.003|

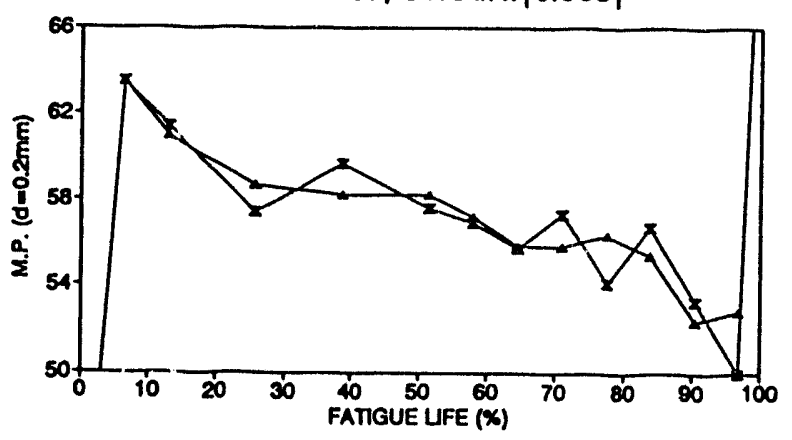

- OBSERVEO DATA - PAOCESSED DATA

Fig. 29 Barkhausen signal as a function of fatigue life at penetration depth of $0.2 \mathrm{~mm}$ for Sample EP07, comparing original data with the data processed by DFT.

Fatigue test: M.P. Measurement SAMPLE EP07; STRAIN:|0.003|

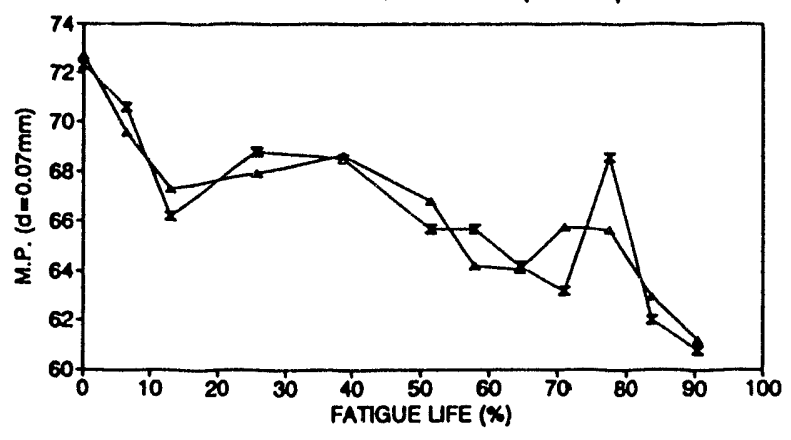

- OBSERVED DATA - PROCESSED DATA

Fig. 30 Barkhausen signal as a function of fatigue life at penetration depth of $0.07 \mathrm{~mm}$ for Sample EP07, comparing original data with the data processed by DFT.
During Stage I (first 10\% fatigue life), there was an increase in Barkhausen amplitude. This increase is a result of fatigue softening caused by breaking up of dislocation tangles during cyclic loading. Therefore discontinuous changes in magnetization (which comprise the Barkhausen signal), will become larger due to an increase in the amplitude of the dromain wall jumps between pinning sites.

In Stage II (20-90\% fatigue life), the Barkhausen signal decreased continuously. This may be due to the movement of dislocations towards the surface. As surface dislocation density becomes higher, the domain walls can experience smaller jumps between two pinning sites, therefore smaller changes in discontinuous magnetization may be expected, leading to small Barkhausen signals.

In Stage III (the last $10 \%$ fatigue life), the Barkhausen signal amplitude increased rapidly with the number of stress cycles as a result of macrocrack formation. When the macrocracks appeared at the surface, several effects can occur, for example, the formation of closure domains at the surface of crack. All these factors can be considered as sources of change in the Barkhausen signal amplitude.

The test results implied that it is possible to predict fatigue failure even before Stage III if the behavior of the Barkhausen signal can be well characterized by the degree of surface damage. However due to the stochastic motion of domain walls the repeatability of the Barkhausen signal results was not very good. (Only 3 out of 5 samples showed the same trend in Barkhausen signal.)

\subsection{Comparison of Magnetic Hysteresis Measurement and Barkhausen Effect Measurement}

The magnetic hysteresis technique, under dc conditions, measures bulk magnetic properties. The magnetic hysteresis parameters, such as coercivity, remanence, initial permeability and maximum differential permeability are sensitive to microstructures and bulk stress, but represent an 
average over the volume of measurement. The magnetic hysteresis measurement data was repeatable with small fluctuations. The test results were easy to interpret and could reliably detect the onset of macrocrack propagation.

Barkhausen effect technique, on the other hand, is a surface sensitive technique. It is sensitive to the surface condition of material and represents properties in typically $0.2 \mathrm{~mm}$ of the material closest to the surface. By monitoring the surface degradation caused by the fatigue damage, it is possible to predict fatigue failure before the propagation of a macrocrack. This has advantages over hysteresis measurements under some test conditions. However, due to the random nature of the Barkhausen emissions, the observed Barkhausen amplitude exhibited considerable variability (therefore advanced signal processing techniques should be used to reduce the noise component), and the repeatability of the measurements was nont on ond as hysteresis measurements. In:crpretation of Barkhausen results is also very difficult. at this time.

In our fatigue test described above, both techniques were found useful in detecting fatigue damage. Each also has some advantages. If we can properly combine these two techniques, it should be possible to predict the remaining life in the materials more precisely.

\subsection{Metallographic Studies}

In order to correlate the surface damage with Barkhausen signals, metallographic studies were performed by inspecting the surface condition of specimens under SEM. Three polished samples were fatigued at constant strain amplitude of C.003. Surface replication was used to inspect the surface condition of the first two samples. The objective was to detect surface damage, such as slip band formation or surface roughening. But the results were not completely satisfactory. Therefore, for the third specimen, we inspected the surface condition directly under the SEM by removing the sample from the MTS machine at predetermined cycles throughout the fatigue life.

In this latter case, there was surface roughening during the fatigue process. However surface modification is not very critical for the formation of microcracks. In fact, the microcracks were initiated mainly at inclusions, such as MnS which is brittle compared with the matrix. These microcracks were observed at least as early as $40 \%$ of expended fatigue life, and later one of them grew continuously to form a macrocrack which led to final mechanical failure. The progress of crack initiation and growth are shown in Figs. 31 - 34.

Since inclusions played an important role in microcrack initiation, the configuration and morphology of these inclusions were of great interest. Two samples were mechanically polished and examined under an optical microscope. It was found that the distribution of inclusions was inhomogeneous as shown in Figs. 35 - 38. This explains why although most specimens exhibited crack initiation at the surface during fatigue, there was a case in which the load began to drop and no surface crack was observed. In that case, the crack was initiated at an inclusion inside the sample and then grew outward.

\subsection{Load-Controlled Fatigue Test}

Load-controlled fatigue tests were conducted at different stress amplitudes, mainly in the high cycle fatigue region. Magnetic properties were measured at pre-determined numbers of fatigue cycles throughout the expended fatigue life. Test results at a stress level of $272 \mathrm{MPa}$ are shown in Figs. $39-40$. It was found that under stress control the magnetic properties changed continuously even after initial fatigue softening. This result was different from the results determined under strain control. Coercivity appeared to be the most useful parameter for monitoring the progress of fatigue. 


\section{Results}
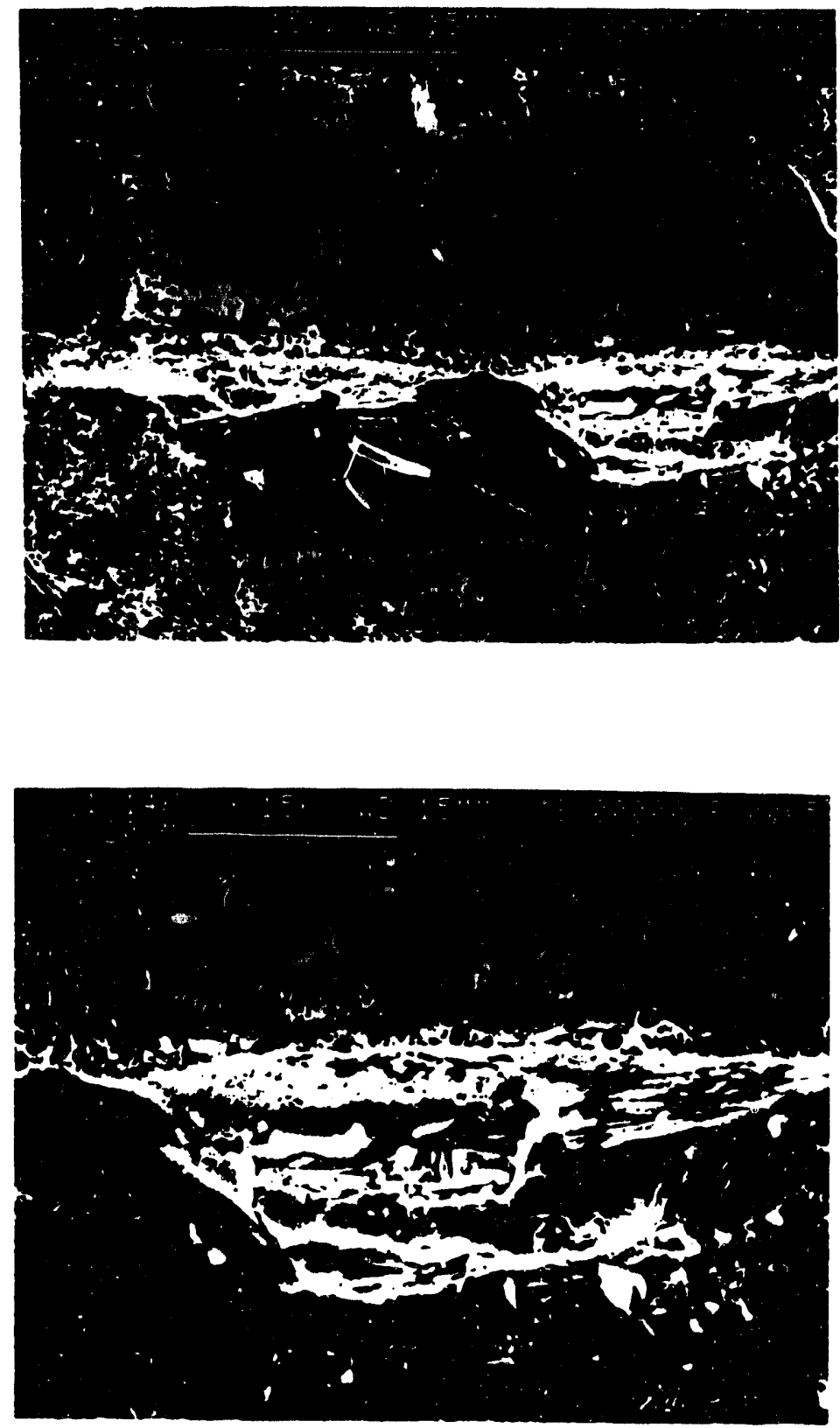

Fig. 31 Microcrack initiated inside the brittle inclusion at about $40 \%$ of fatigue life. 
Results
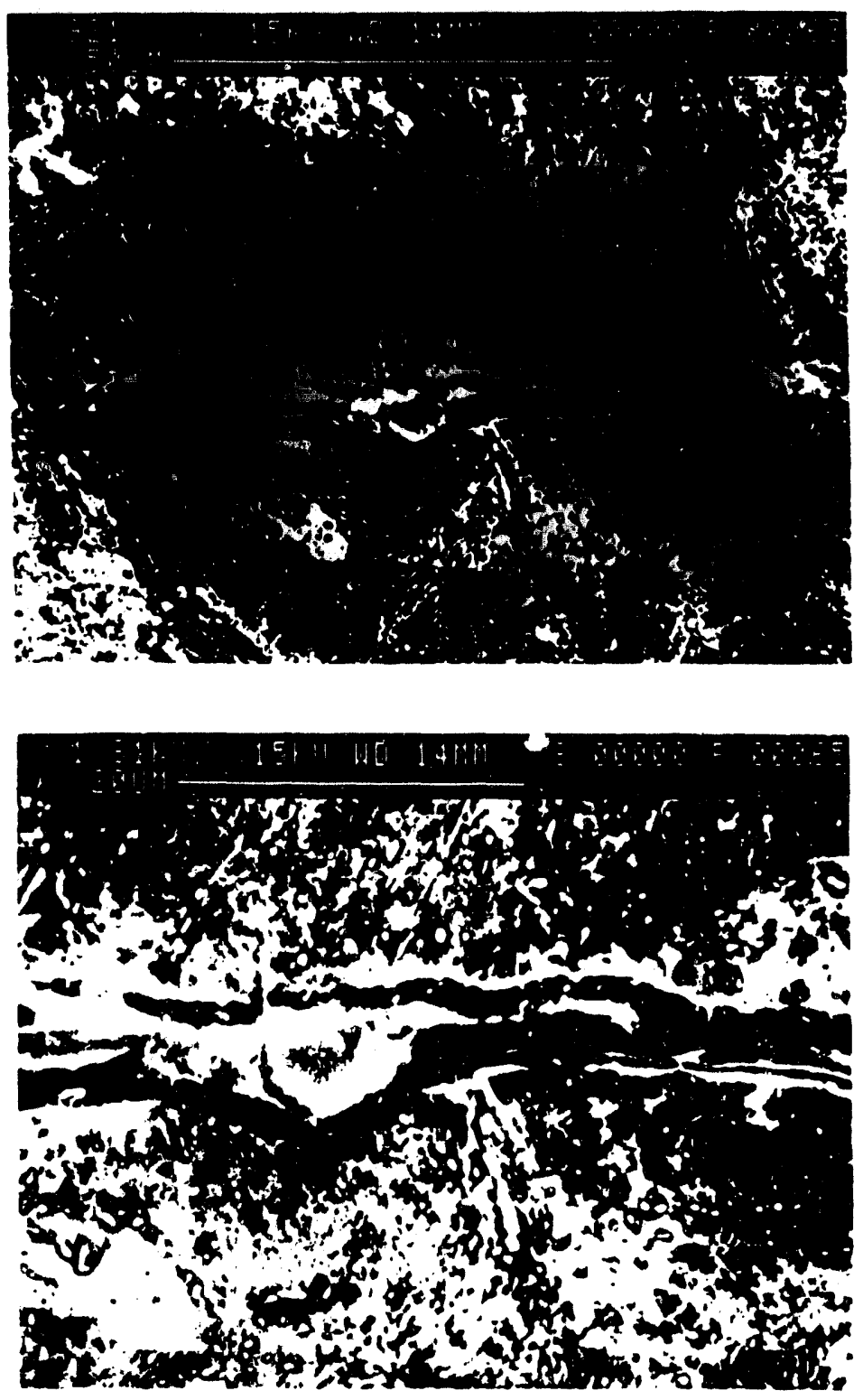

Fig. 32 Microcrack initiated at the edge of brittle inclusion at about $40 \%$ of fatigue life. 


\section{Results}
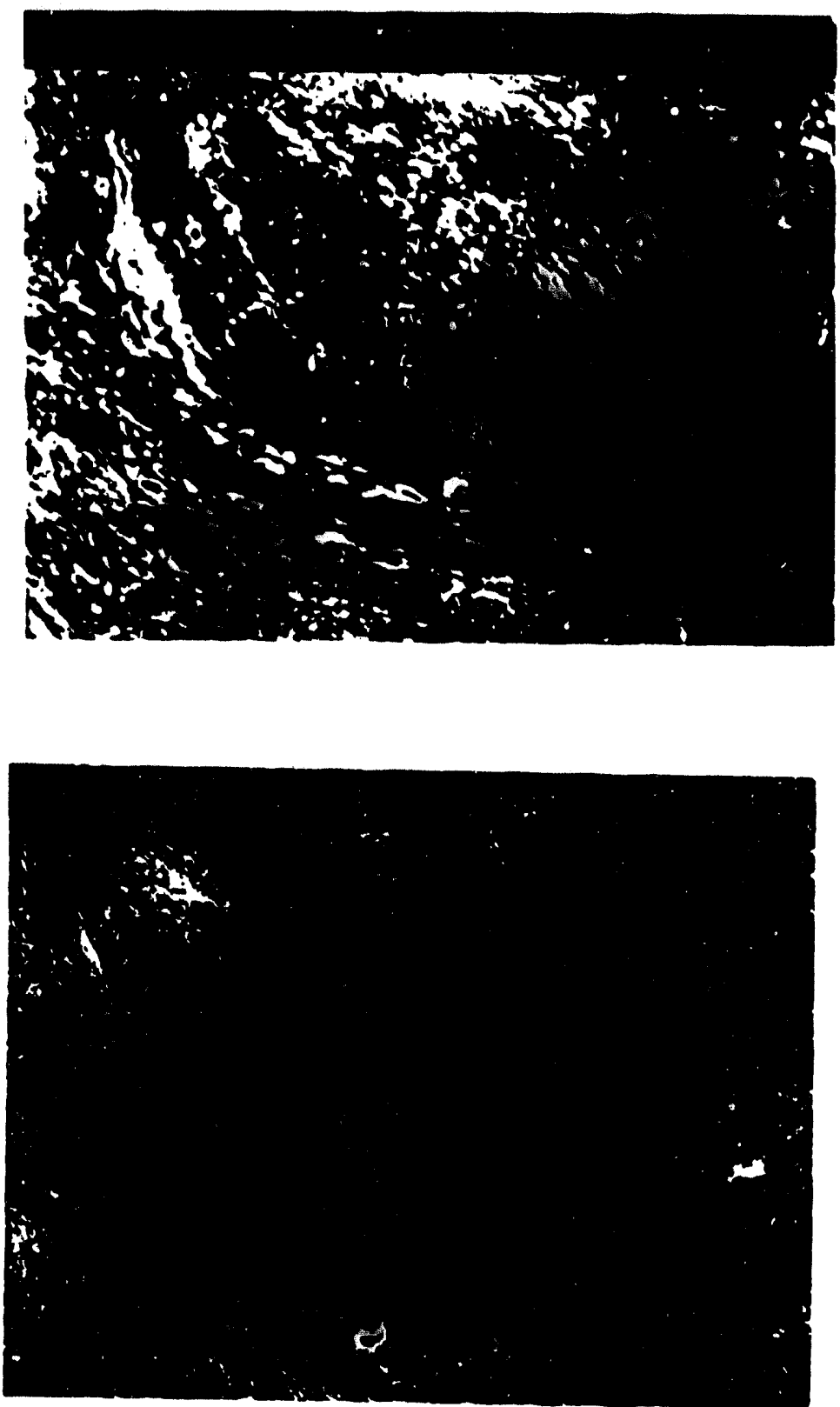

Fig. 33 Growth of microcrack due to fatigue at about $60 \%$ of fatigue life. 
Results

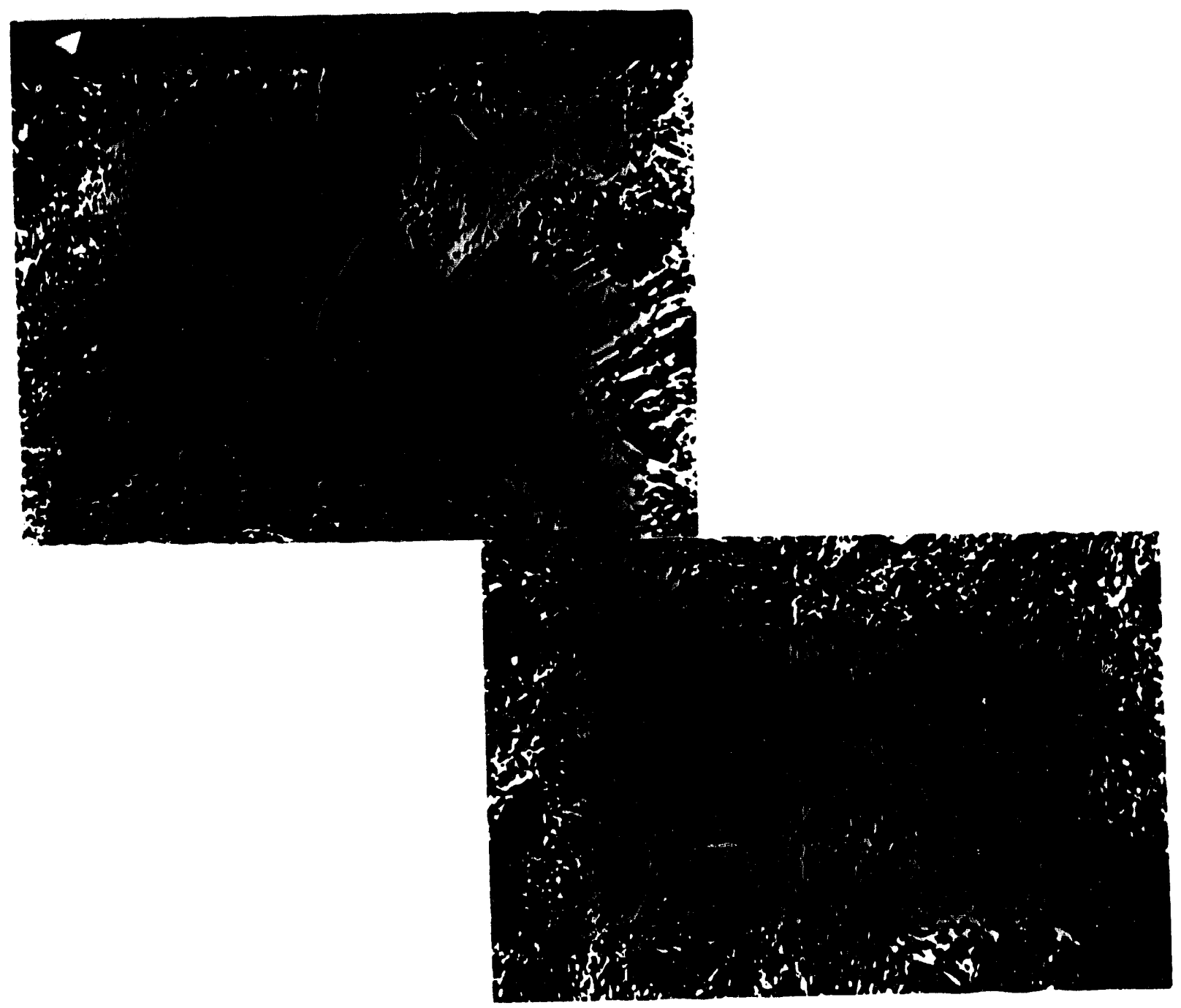

Fig. 34 Growth of microcrack due to fatigue at about $70 \%$ of fatigue life. 


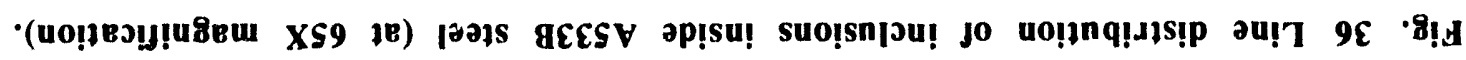

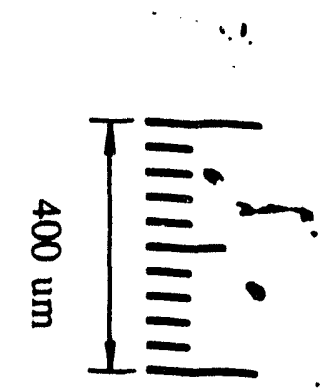

-

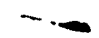

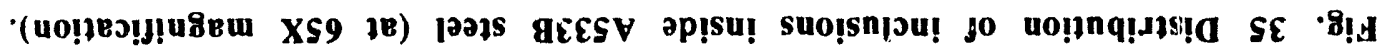

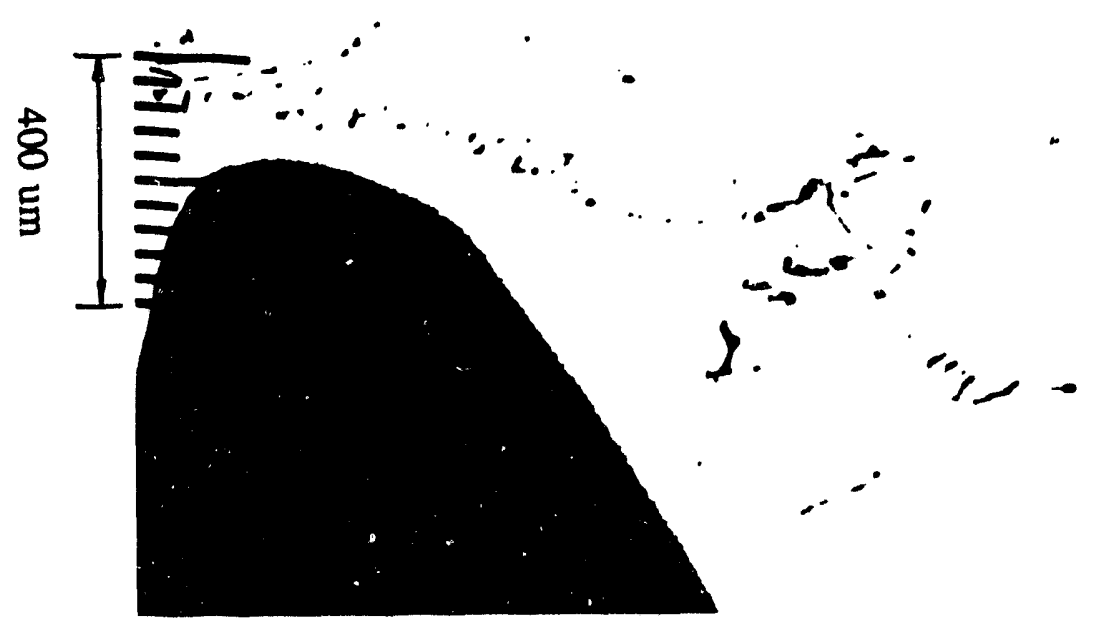


Results

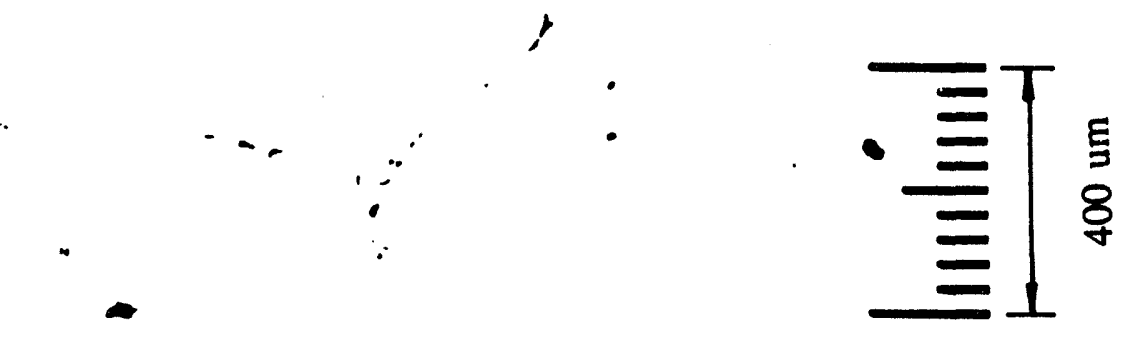

Fig. 37 Distribution of inclusions on grain boundary inside A533B steel (at 65X magnification).

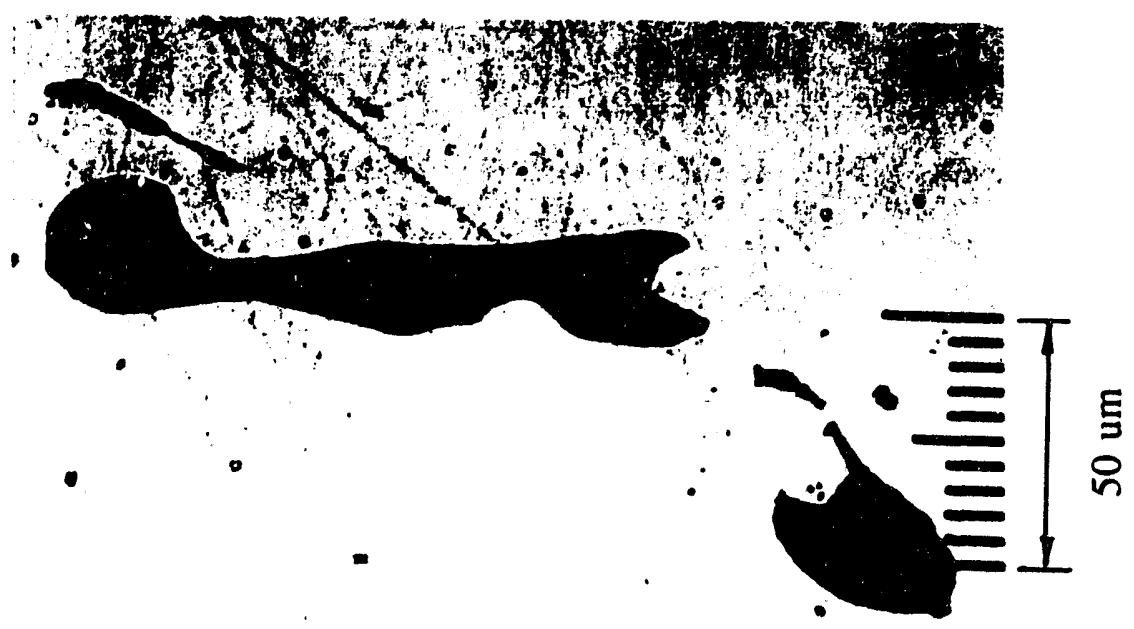

6

Fig. 38 Shape of inclusion (at 520X magnification). 


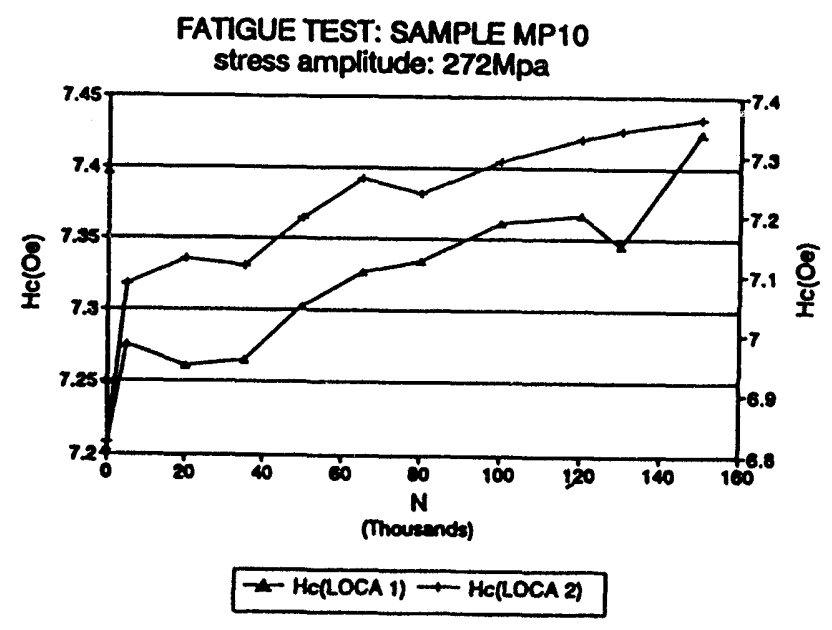

Fig. 39 Variation of coercivity at two different locations with expended fatigue life for Sample MP10 under fatigue test with stress amplitude of $272 \mathrm{MPa}$.

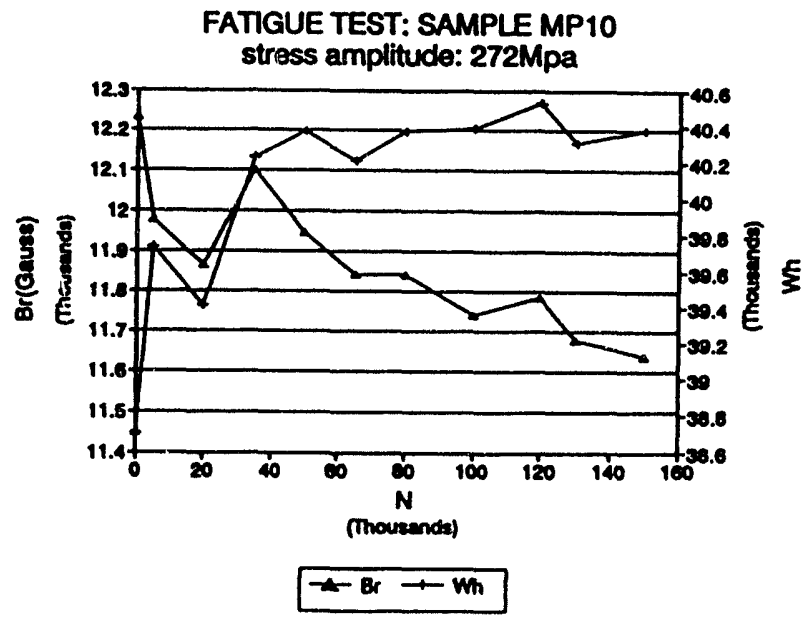

Fig. 40 Variation of remanence and hysteresis loss with expended fatigue life for Sample MP10 under fatigue test with stress amplitude of $272 \mathrm{MPa}$.
Further fatigue tests showed that during loadcontrolled fatigue tests, the maximum tensile and compressive strain accumulated linearly with the logarithm of fatigue cycle. The results are shown in Figs. 41- 42. Based on the fact that the plastic strain is often linearly related to the coercivity of the material $[15,18]$, at least for a small region in monotonic stress loading, a phenomenological relationship was developed to correlate the number of stress cycles with coercivity. The equation predicted a linear relationship between the coercivity and the logarithm of the number of fatigue cycles. Two more specimens were fatigued under constant load amplitude, and magnetic measurements were taken at predetermined intervals chosen according to the logarithm of the number of fatigue cycles. Results are shown in Figs. 43 - 44. These matched a simple phenomenological relationship described by the equations in Appendix Al.6, showing a direct correlation between the magnetic properties (e.g. Hc) and the logarithm of N.

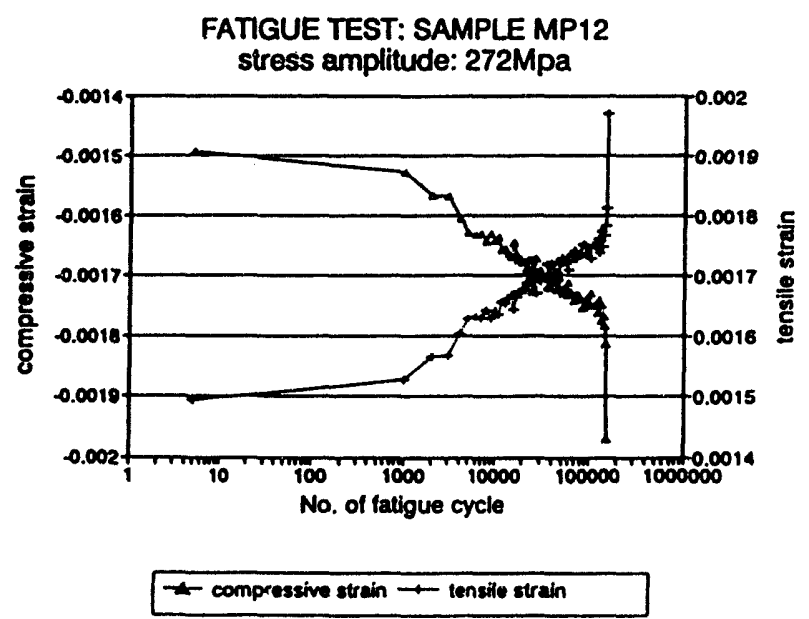

Fig. 41 Variation of compressive and tensile strain with expended fatigue life for Sample MP12 under fatigue test with stress amplitude of $272 \mathrm{MPa}$. 
Sample Mp13

Stress amplitude: $243 \mathrm{Mpa}$

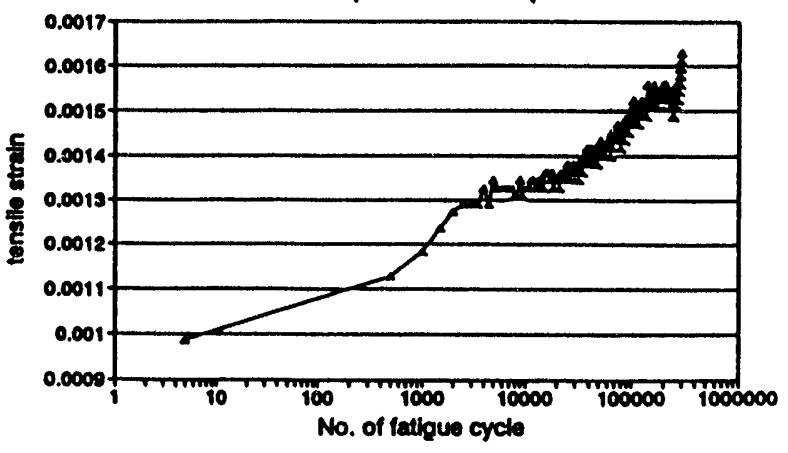

- tonslle strin

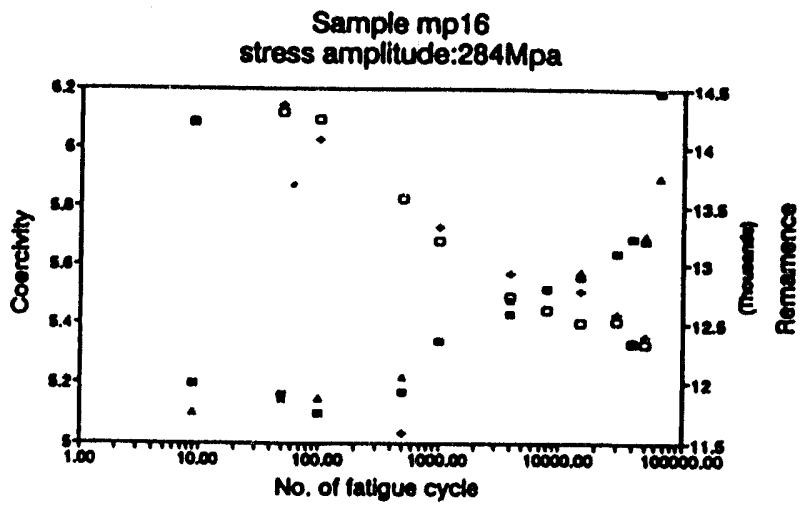

- Hetocal a Hetocan + Brtocal O Brtocan

Fig. 44 Variation of coercivity and remanence with expended fatigue life for Sample MP16 under fatigue test with stress amplitude of $284 \mathrm{MPa}$.

It was evident that the A533B material underwent fatigue softening when cyclically stressed. This was demonstrated for example when under the strain-controlled fatigue process, the load dropped significantly during the first a few hundred cycles. However, under the load-controlled fatigue process, the coercivity increased, which implied that magnetic hardening occurred. Usually magnetic hardening is also accompanied by mechanical hardening. So the observation that the material underwent a mechanical softening accompanied by a magnetic hardening was unexpected. In fact this seemingly contradictory phenomenon can be explained by the Bauschinger effect. When A533B material was fatigued, additional dislocations were generated which acted as pinning sites for domain wall motion, thus increasing the coercivity. However, during the cyclic stress, dislocations piled up at grain boundaries or precipitates, forming dislocation tangles. With reversed loading, the dislocations at the trailing edges of the tangles moved away from the grain boundaries and precipitates more easily, causing an apparent softening of the mechanical properties. 
Although the detailed mechanism for the relationship between the coercivity and the number of fatigue cycles is not clear, the phenomenological relation found in this project gives a useful equation for applying magnetic NDE to fatigue detection. One of the significant results of this relationship is that, for the first time, the number of stress cycles was shown to be related directly to a measurable magnetic parameter. Therefore the measurement of coercivity may provide a way to estimate fatigue damage accumulation. At this point, the advantage of magnetic hysteresis measurement techniques over the Barkhausen effect technique is clear, since the magnetic hysteresis technique, can estimate the number of fatigue cycles a specimen has experienced, and the measurements of hysteresis parameters are more reproducible.

\subsection{Prestrain Fatigue Test}

Since fatigue life and magnetic properties depend on the initial state of the material, the role of stress history (i.e., residual stress or any previous dislocation substructure due to the plastic straining) on fatigue testing parameters was examined. Five specimens were prestrained to different strain level from 0.008 to 0.021 . Fatigue tests and magnetic measurements were performed under different fatigue conditions. Test results are shown in Figs. 45 - 48. It can be seen that in both strain-controlled and load-controlled fatigue processes, the coercivity and hysteresis loss decreased and the remanence increased. In the strain-controlled fatigue tests, the load also showed a decrease during the fatigue life which implied fatigue softening. All these results can be explained by the fact that a large amount of dislocations were created by the initial plastic deformation. When the specimens were cyclically stressed, dislocations recombined as well as accumulating on the grain boundary and precipitates, resulting in a decrease in dislocation density and therefore leading to both mechanical softening and magnetic softening.

The test results again supported the suggestion that the decrease in coercivity was dependent on the logarithm of number of fatigue cycles. This was in agreement with the result that the logarithm of the number of fatigue cycle determined the accumulation of fatigue damage in these materials. NUREG/GR-0013

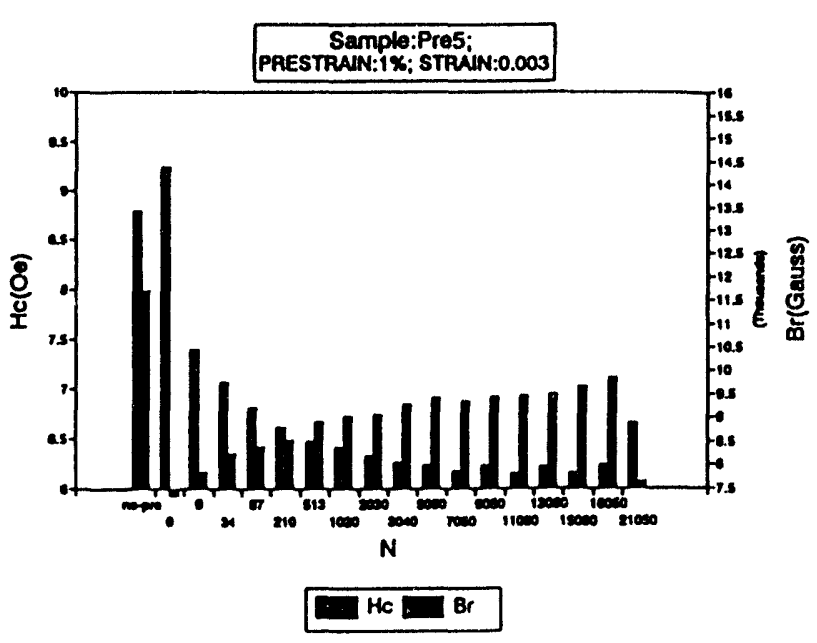

Fig. 45 Variation of cocrcivity and remanence with expended fatigue life for Sample PRE5 under fatigue test with stress amplitude of 0.003 . The Sample was prestrained with residue strain of 0.01 .

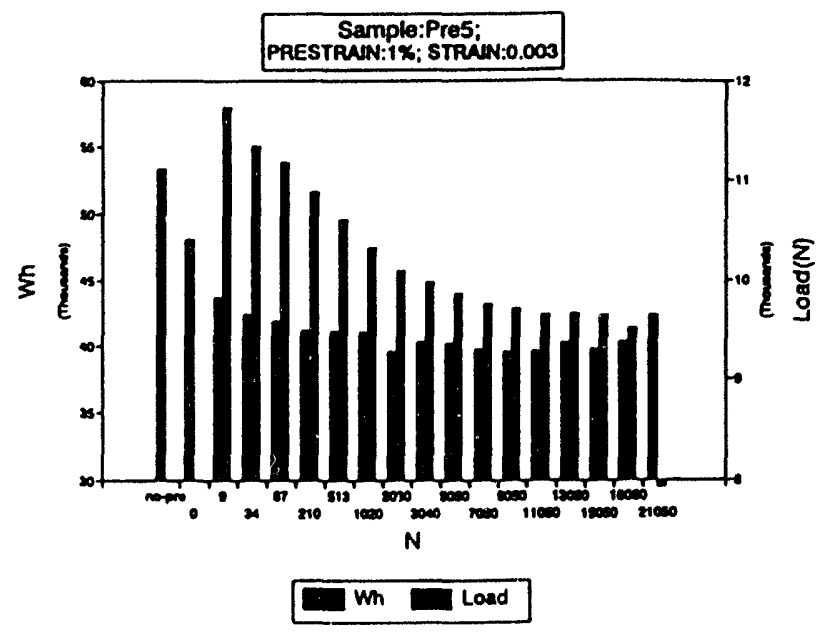

Fig. 46 Variation of hysteresis loss and tensile load with expended fatigue life for Sample PRE5 under fatigue test with stress amplitude of 0.003 . The specimen was prestrained with residue strain of 0.01 . 


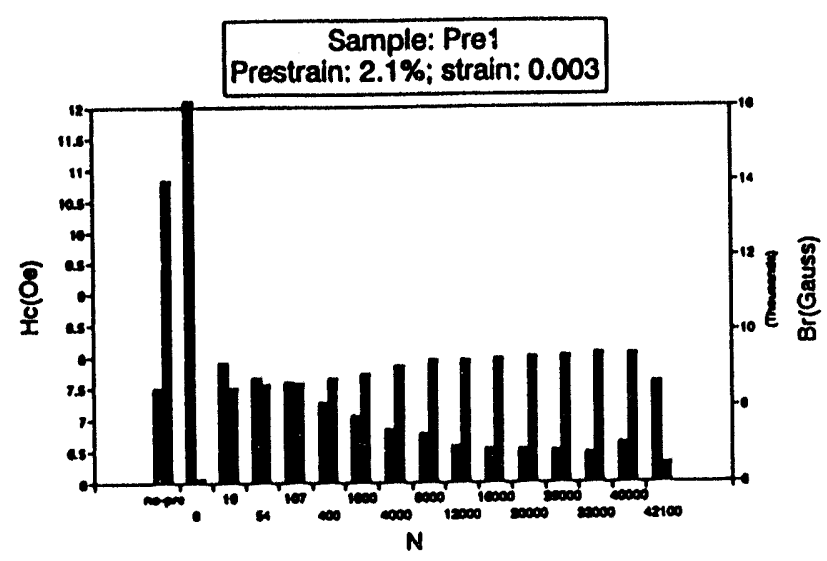

$\mathrm{Hc} B \mathrm{Br}$

Fig. 47 Variation of coercivity and hysteresis loss with expended fatigue life for Sample PREI under fatigue test with stress amplitude of 0.003 . The specimen was prestrained with residue strain of 0.021 .

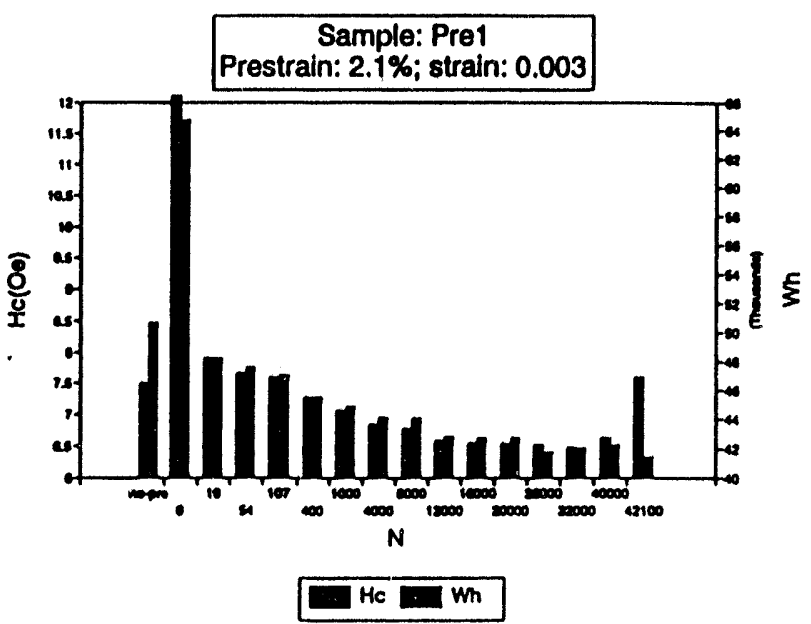

Fig. 48 Variation of coercivity and remanence with expended fatigue life for Sample PRE1 under fatigue test with stress amplitude of 0.003 . The specimen was prestrained with residue strain of 0.021 . 


\section{Conclusions}

\subsection{Summary of Progress}

The project has progressed on schedule throughout. A533B steel specimens were acquired from Oak Ridge National Laboratory and they were machined into tensile test specimens for laboratory tests. High cycle and low cycle fatigue measurements were made on both unprestrained and prestrained specimens. Magnetic measurements in the form of hysteresis curves were taken on these specimens throughout their fatigue life. These measurements were analyzed to give the main magnetic properties of coercivity, remanence, hysteresis loss and maximum differential permeability, which showed changes with expended fatigue life. These changes were apparent particularly in the early stages of fatigue (as a result of fatigue softening) and in the final stages of fatigue (as a result of crack growth) in the strain-controlled fatigue tests. In the loadcontrolled fatigue tests, the magnetic properties changed continuously with expended fatigue life.

A new set of software routines has been written to enable the tensile test machine to be controlled by computer and to ensure that the fatigue tests begin with stress in a particular direction and end with completion of a full fatigue cycle. This eased the task of making measurements by automating the whole process and guaranteed that fatigue cycling terminated under the same external mechanical condition.

A commercial Barkhausen inspection system was acquired from American Stress Technologies. This device has the capability of being calibrated for applied stress. The measurements can be performed at different detection frequencies and hence at different depths in the specimens. Since a correlation between magnetic parameters and fatigue damage has been observed in this work, we have also investigated whether there is a similar correlation between Barkhausen measurements and fatigue damage. The results have shown that the Barkhausen signal amplitude is sensitive to accumulated fatigue damage, and that therefore these measurements have some potential for nondestructive evaluation of fatigue.
A relation has been observed between the magnetic coercivity and the number of fatigue cycles based on the test results under load-controlled fatigue. The relation shows that the coercivity is proportional to the logarithm of the number of fatigue cycles. This is the first time that an empirical relation has been found which relates fatigue damage to magnetic properties.

\subsection{Detection of Volumetric Properties Versus Localized Flaws}

Results have shown that in order to detect localized flaws the magnetic field sensor (Hall probe) must be within $1 \mathrm{~mm}$ of the location of the flaw. Therefore most changes that are detected by the hysteresis method are actually volumetric changes in magnetic properties rather than highly localized changes due to cracks. This is a significant advantage because it is then not necessary to be directly above the fatigue crack order to detect impending failure.

The Barkhausen method has been used to evaluate the surface condition of the material at depths of $0.02,0.07,0.2 \mathrm{~mm}$. This technique gives complementary information to the magnetic hysteresis measurements, and has also been found to be affected by impending fatigue failure. It is widely believed that the condition of the material surface is very significant in determining the remaining fatigue life.

\subsection{Detection of Strain-Controlled Fatigue Versus Load- Controlled Fatigue}

Strain-controlled fatigue tests were mainly conducted in the low cycle fatigue regime, while load-controlled fatigue tests were conducted in the high cycle regime. In the strain-controlled fatigue tests, magnetic properties changed markedly at the beginning and at the end of fatigue life. This is believed to be due to fatigue softening and stable microcrack propagation respectively. On the other hand, in the load-controlled fatigue tests, magnetic 
properties were observed to change continuously during the whole fatigue life.

The differer $x$ between the behavior of the magnetic properties during two different types of fatigue test condition can be explained. In the strain-controlled test condition, after initial fatigue softening, the mechanical properties of material stabilized. Therefore no significant variations in magnetic properties were observed during $10-80 \%$ fatigue life. On the other hand, in load-controlled test conditions microcracks which reduced the effective cross section of tensile specimen can result in a progressively higher stress amplitude from one cycle to the next. This generates extra dislocations that can pin domain walls. Therefore in load-controlled fatigue tests, coercivity increased with the number of fatigue cycles.

In the prestrained fatigue tests, because of the large amount of initial dislocations generated by plastic deformation, the recombination and the sweeping away of the dislocations to the grain boundary dominated over dislocation generation. Therefore, in both load-controlled and straincontrolled fatigue tests, the bulk dislocation density decreased and resulted in the coercivity decreasing with the number of fatigue cycles.

\subsection{Prognosis}

Magnetic inspection methods are a very promising technique for nondestructive evaluation of such problems as mechanical degradation in steels. In this work it has been shown that under straincontrolled fatigue tests, drastic changes occurred in coercivity and other magnetic hysteresis parameters during the last $10 \%$ of fatigue life. Under load-controlled fatigue conditions, coercivity increased linearly throughout the fatigue life with the logarithm of the number of fatigue cycles. If the material was sufficiently prestrained, then coercivity decreased linearly with the logarithm of the number fatigue cycles. Although the Barkhausen effect also showed sensitivity to expended fatigue life, as shown in the results here, the signal was quite noisy and the repeatability was not entirely satisfactory. Besides, it was extremely difficult to explain the observed changes in the Barkhausen signals. On the other hand the magnetization, as embodied in the hysteresis curve, can be interpreted in terms of domain wall/defect interactions in steel, and this therefore lends itself to a much simpler interpretation.

There are a few models of ferromagnetic hysteresis in use at present, although for this particular investigation we have used the model developed by one of the authors [20]. In this way we were able to circumvent the difficulties which have hindered earlier investigations in this area. These have often observed a high sensitivity of magnetic signatures to stress (e.g., Barkhausen or magnetoacoustic signals) but have been unable to interpret them. The present work has some distinct advantages over earlier investigations because of the opportunity afforded to interpret the resuits in terms of underlying mechanisms through the hysteresis model.

For example in the case of fatigue damage, it is known that in the early stages of the fatigue process the dislocation density changes rapidly. Magnetic domain walls are impeded by dislocations and, according to the theoretical model developed for hysteresis, this leads to a change in coercivity. Such a change in coercivity is indeed observed in practice. These verifications of the model lend confidence to our use of it in solving complex problems based on hysteresis measurements, such as the evaluation of expended fatigue life. 


\section{References}

1. Jiles, D. C., S. Hariharan and M. K. Devine, "Magnescope: a portable magnetic inspection system for evaluation of steel structures and components," IEEE Trans. Mag., 26:2577.

2. Tiitto, K., and S. Tiitto, American Stress Technologies Inc., 61 McMurray Rd., Pittsburgh, PA 15241.

3. Jiles, D. C., "Review of magnetic methods for NDE," NDT International, 21:311.

4. Jiles, D. C., "Review of magnetic methods for NDE (part 2)," NDT International, 23:83.

5. Theiner, W. A., and I. Altpeter "Determination of residual stresses using micromagnetic parameters" in New Procedures in NDT, edited by P.Holler, SpringerVerlag, 1983.

6. Tiitto, K., "Use of Barkhausen noise in fatigue," Nondestructive Testing and Evaluation, 5:27.

7. Birkett, A. J., W. D. Corner, B. K. Tanner and S. M. Thompson, "Influence of plastic deformation on Barkhausen power spectra in steels," J. Phys. D. (Appl. Phys.), 22:1240.

8. Buttle, D. J., G. A. D. Briggs, J. P. Jakubovics, E. A. Little and C. B. Scruby, "Magnetoacoustic and Barkhausen emission in ferromagnetic materials," Phil. Trans. Roy. Soc., A320:363.

9. Buttle, D. J., J. P. Jakubovics, G. A. D. Briggs and C. B. Scruby, "Magnetoacoustic and Barkhausen emission from domain wall interactions with precipitates in Incoloy-940," Phil. Mag., 55:735.

10. Scruby, C. B., D. J. Buttle, G. A. D. Briggs and J. P. Jakubovics, "The monitoring of stress and microstructure by magnetoacoustic emission," U.K. Atomic Energy Authority Report No. AERE-R-12781$2,1987$.
11. Langman, R., "Measurement of the mechanical stress in steel by means of rotation of magnetic field strength," NDT International, 14:255.

12. Jiles, D. C., and D. L. Atherton, "Theory of the magnetization process in ferromagnets and its application to the magnetomechanical effect," J.Phys. D. (Appl. Phys.), 17:1265.

13. Atherton, D. L., L. W. Coathup, D. C. Jiles, L. Longo, C. Welbom and Teitsma, "Stress induced magnetization changes in steel pipes," IEEE Trans. Mag., Mag-19:1564 and MAG-20:2129.

14. Atherton, D. L., and D. C. Jiles, "Effects of stress on magnetization," NDT International, 19:15.

15. Jiles, D. C., "The effect of residual strain on the magnetic properties of AISI 4130 steels with various microstructures," J. Phys. D (Appl. Phys.), 21:1196.

16. Jiles, D. C., "Creep damage assessment of Cr-Mo steels using magnetic inspection," Contract report prepared for Ontario Hydro, Research Division, Nov. 1991.

17. Govindaraju, M. R., A. Strom, D. C. Jiles, S. B. Biner, Z. J. Chen, "Evaluation of fatigue damage in steel structural components by magnetoelastic Barkhausen signal analysis," J. Appl. Phys., 73(10):6165.

18. Jiles, D. C., "Variation of the magnetic properties of AISI 4140 steels with plastic strain," Phys. Stat. Sol. (a), 108:417.

19. Jiles, D. C., "Magnetic properties and microstructure of AISI 1000 series carbon steel," J. Phys. D (Applied Physics), 21:1186.

20. Jiles, D. C., and D. L. Atherton, "Theory of ferromagnetic hysteresis," J. Mag. Mag. Mater., 61:48. 


\section{Appendix 1: Papers Published as a Result of this Work}

1) "Measurements of magnetic circuit characteristics for comprehension of intrinsic magnetic properties of materials from surface inspection", Z. J. Chen, M. K. Devine, D. C. Jiles. J. Appl. Phys. 73(10), 7p. 56205622, 15 May, 1993. (Refereed by journal editorial board.)

2) "Analytic model calculation of magnetic field in a magnetic half space due to surface magnetic charge", Z. J. Chen, M. R. Govindaraju, D. C. Jiles. J. Appl. Phys. 73(10), pp. 6858-6860, May 1993. (Refereed by journal editorial board.)

3) "Evaluation of fatigue damage in steel structural components by magnetoelastic Barkhausen signal analysis", M. R.

Govindaraju, A. Strom, D. C. Jiles, S. B.

Biner, Z. J. Chen. J. Appl. Phys. 73(10), pp. 6165-6167, 15 May 1993. (Refereed by journal editorial board.)
4) "Micromagnetic surface measurements for evaluation of surface modification due to cyclic stress", Z. J. Chen, A. Strom, D. C. Jiles. IEEE Magnetics Transactions 29, pp. 30313033, 1993. (Refereed by journal editorial board.)

5) "Modelling of reversible domain wall motion under the action of magnetic field and localized defects", Z. J. Chen, D. C. Jiles. IEEE Magnetics Transactions 29, pp. 2554-2556, 1993. (Refereed by journal editorial board.)

6) "Estimation of fatigue exposion from magnetic hysteresis parameters", Z. J. Chen, J. Kameda, D. C. Jiles. Accepted for publication in J. Appl. Physics. (Refereed by journal editorial board.) 


\section{Appendix 2: Conference Presentations}

37th Annual Conference on Magnetism and Magnetic Materials, Houston, December 1-4, 1992.

1) "Measurements of magnetic circuit characteristics for comprehension of intrinsic magnetic properties of materials from surface inspection." (Refereed by conference committee.)

2) "Analytic model calculation of magnetic field in a magnetic half space due to surface magnetic charge." (Refereed by conference committee.)

International Magnetics Conference, Stockholm, Sweden, April, 1993.

3) "Micromagnetic surface measurements for evaluation of surface modification due to cyclic stress." (Refereed by conference committee.)

4) "Modelling of reversible domain wall motion under the action of magnetic field and localized defects." (Refereed by conference committee.) 38th Annual Conference on Magnetism and Magnetic Materials, Minneapolis, November 15 18, 1993.

5) "Measurements of intrinsic magnetic properties from surface inspection", Z.J. Chen, M. Negley, D.C. Jiles. Presented at the 38th Annual Conference on Magnetism and Magnetic Materials, Minneapolis, Nov. 15$18,1993$.

ASM Materials Week Conference, Pittsburgh, October 18-21, 1993.

6) "SEM \& TEM investigation of fatigue induced microstructural changes and resulting effect on magnetic properties of structural steels," M. R. Govindaraju, Z. J. Chen, A. Strom, S. B. Biner, D. C. Jiles. To be presented at 1993 ASM Materials Week, Pittsburgh, Pennsylvania, Oct. 18-21, 1993. 


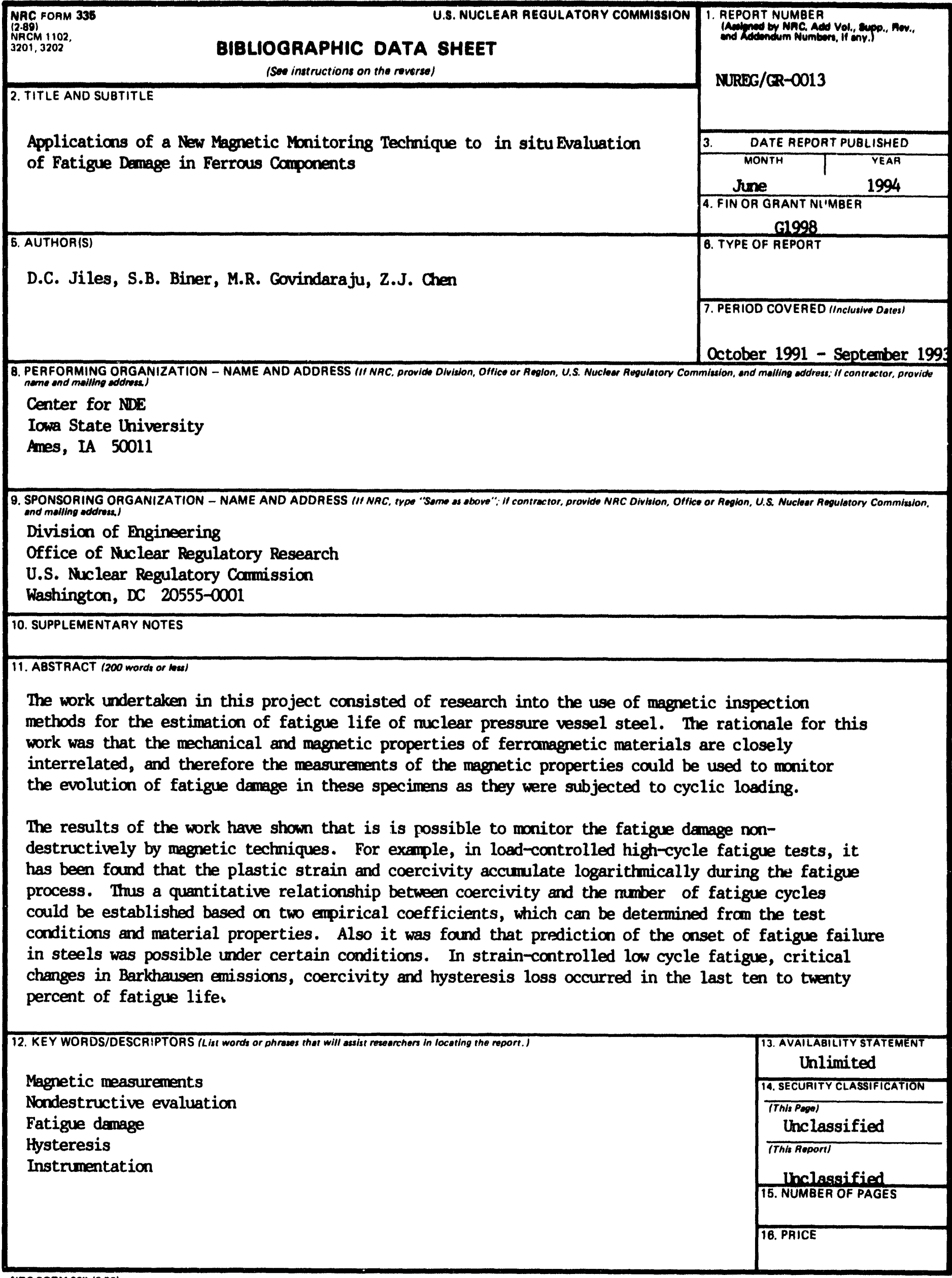



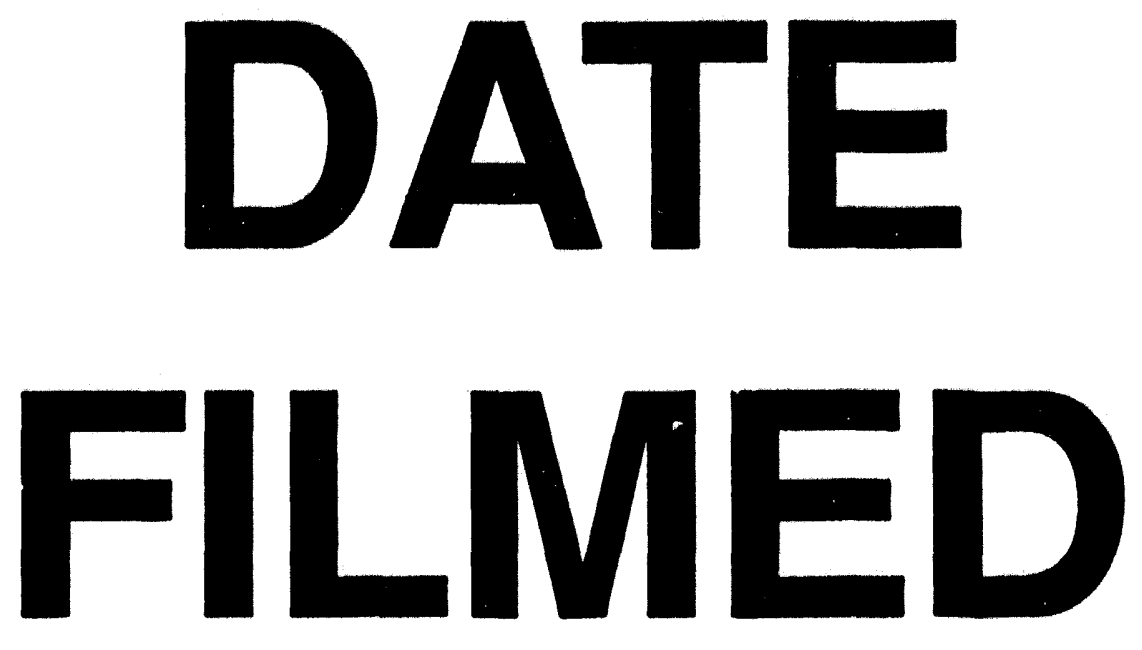

$8 / 22 / 94$
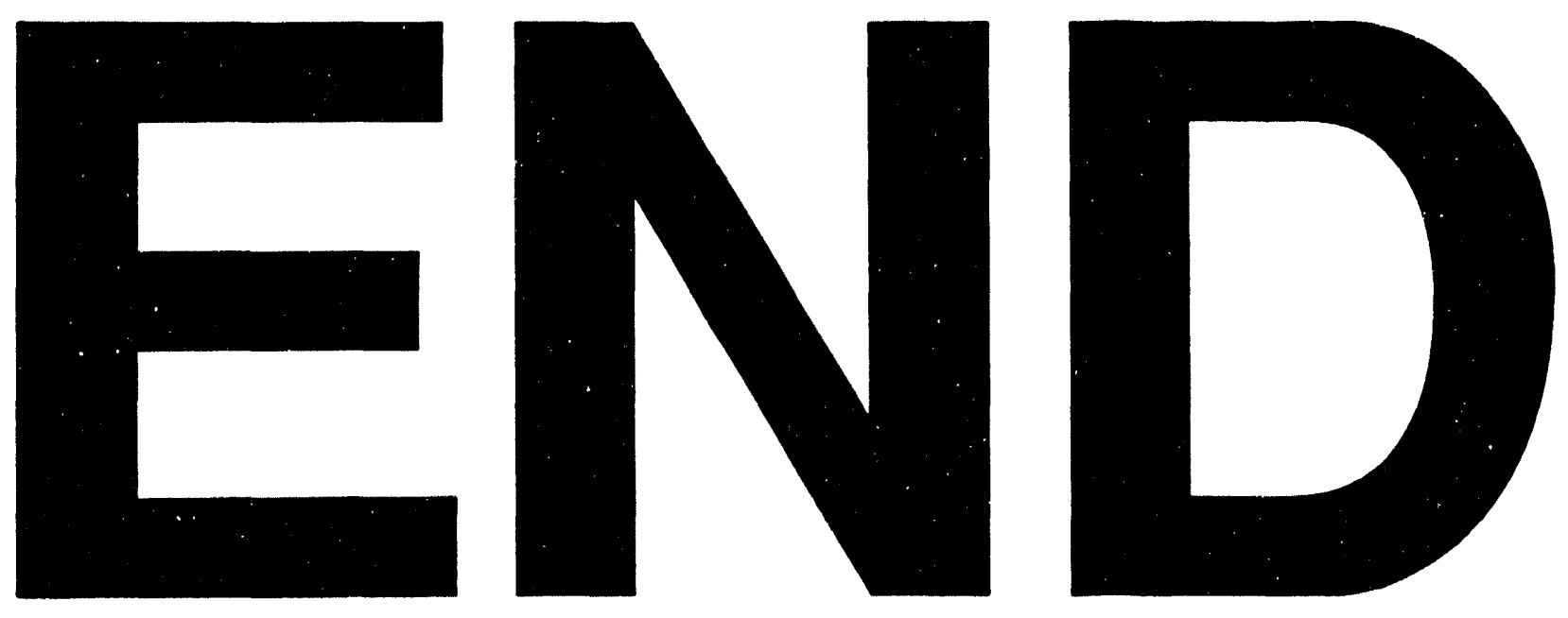
